\title{
2019 Vestibular Oriented Research Meeting May 19-22, 2019 \\ Hosted by The Ohio State University Wexner Medical Center at the Marriott at the University of Dayton
}

\section{Quantifying the links between age, vestibular function, and balance}

\section{$\underline{\text { (Abstract 1, Poster Location S) }}$}

Maria Carolina Bermúdez Rey, MD and Faisal Karmali, Ph.D.

Jenks Vestibular Physiology Lab, MEEI; Harvard Medical School Boston MA

Torin Clark, Ph.D.

University of Colorado - Boulder, Boulder CO

Sinem Balta Beylergil, PhD

Case Western Reserve University, Cleveland $\mathrm{OH}$

Daniel M. Merfeld, Ph.D.

The Ohio State University, Columbus $\mathrm{OH}$ and

Naval Medical Research Unit - Dayton $\mathrm{OH}$

Wei Wang, Ph.D.

Division of Sleep Medicine, Brigham

and Women's Hospital, Boston, MA

Both vestibular function and balance degrade with age, and balance degradation contributes to falls. While multiple causes contribute to both balance declines and falls, there have been few comprehensive empirical investigations focused on the effect of aging on vestibular function and on the specific vestibular contributions to balance that mediate (i.e., explain a significant fraction of) the effect of age on balance. Our goals were to quantify age-effects on vestibular function, to determine if vestibular function significantly mediates the effect of age on balance, and to quantify the fraction of any such statistically significant age-effect on balance. Balance was quantified as complete/incomplete on a standard Romberg 4-condition foam balance test for 99 subjects. Vestibular thresholds for 5 motions $(0.2 \mathrm{~Hz}$ roll tilt and $1 \mathrm{~Hz}$ roll tilt, yaw rotation, y-translation, and z-translation) were determined using standard methods with motion provided by a Moog 6DOF motion platform for 105 subjects. Mediation analyses were performed to determine if any of the five vestibular thresholds we measured mediated the previously reported age-effect on balance. We report: (a) that vestibular thresholds for all 5 different motions increased with age above the age of 40 (1), (b) that the chance of not completing the balance test depended only on roll tilt $0.2 \mathrm{~Hz}$ thresholds and not sex nor any of the other four threshold measures and that the chance of failing condition 4 depends significantly on both $0.2 \mathrm{~Hz}$ roll tilt thresholds (p$0.006)$ and age $(p=0.013)(2)$, and (c) $0.2 \mathrm{~Hz}$ roll tilt thresholds were found to significantly mediate the relationship between age and balance - explaining between $33 \%$ and $55 \%$ of the total age-effect on balance. If these findings are confirmed by future studies, this may provide an opportunity to improve balance (and presumably reduce fall risk) via specific therapies tailored to improve vestibular function.

\section{References}

1. Bermúdez Rey et al. (2016). Frontiers in Neurology 7(162)

2. Karmali et al. (2017). Frontiers in Neurology $8(578)$.

Acknowledgements: Funded by NIH/NIDCD R01DC01458, R01-DC014924, and R03-DC013635.

\section{Performance, learning, retention and training of dynamic stabilization skill with degraded gravitational cues}

\section{(Abstract 2, Poster Location G)}

Vivekanand Pandey Vimal, Ph.D, Paul DiZio, Ph.D, James Lackner, Ph.D

Ashton Graybiel Spatial Orientation Lab, Brandeis University

Our first objective was to compare performance and learning in a dynamic stabilization task in conditions 
where gravitational cues relevant to position were present or absent. Blindfolded subjects were secured into a device programmed with inverted pendulum dynamics and were instructed to use a joystick to stabilize themselves about the balance point [1-4]. When subjects balanced in either the Vertical Roll Plane (VRP) or the Horizontal Yaw Axis, where otolith and somatosensory gravitational cues about tilt were available, they performed the task well and showed robust learning across trials. In contrast, subjects who balanced in the Horizontal Roll Plane (HRP) or the Vertical Yaw Axis, where relevant gravitational cues were absent and only semicircular canal cues about motion were available, repeatedly "crashed" (exceeded angular displacements of $60^{\circ}$ ) and showed minimal learning and a characteristic pattern of positional drifting. This condition has relevance for studying spatial disorientation in military pilots and astronauts. We also found that learning acquired during HRP balancing transferred to VRP balancing but there was no transfer in the other direction, indicating that balance control consists of 2 dissociable processes: aligning with the gravitational vertical using position cues and dynamic stabilization using motion cues. Our second objective was to design a training program that would enhance performance and learning in the HRP where subjects do not have relevant gravitational cues [5]. Because subjects in the HRP rely on motion cues, we created a training paradigm that focused on refining subjects' abilities to dynamically stabilize, independent of aligning with the gravitational vertical by setting the balance point to be different from the gravitational vertical in the VRP. Subjects were not told the location of the balance point and had to ignore biasing positional information from gravitational cues and rely instead on motion cues to determine the location of the balance point. We found that subjects in this training group who were later tested in the HRP showed significant learning across many metrics whereas the control group, who practiced in the HRP for the same number of trials, showed very minimal learning. The training group retained this learning when retested 4 months later.

\section{References}

1. Vimal VP, Lackner JR, P DiZio. EBR (2016)

2. Vimal VP, DiZio P, Lackner JR. EBR (2017)

3. Vimal VP, Lackner JR, Paul DiZio. EBR (2018)

4. Panic AS, DiZio P, Lackner JR. AEROSP MED HUM PERF (2017)

5. Vimal VP, DiZio P, Lackner JR. EBR (submitted)
Acknowledgement: Funded by the Air Force Office of Scientific Research AFOSR FA9550-12-1-0395 and VV is funded by TRISH through Cooperative Agreement NNX16AO69A.

\section{Immersive visual motion stimuli effects on cognitive performance tracking}

\section{(Abstract 3)}

Anil K Raj, MD, Margaret Freyaldenhoven and Sonia Yanovsky

Florida Institute for Human and Machine Cognition

The rapid advancement in aircraft technologies has increased pilot task load, with fewer operators controlling more subsystems. Aircraft sensors and instrumentation can provide real-time feedback regarding the current state and reserve of the aircraft systems, however, no sensors provide similar data about the pilot. Quantification of pilot state using could provide a pathway for pilots to optimize their mission performance and effectiveness while maintaining or improving safety. Incorporation of multimodal psychophysiologic and environmental contextual sensors with machine learning (ML) that identify and display data features that correlate with shifts in cognitive state would allow pilots to proactively adjust tactics in a closed-loop, real-time fashion. Our goal in this study was to determine how a full field of view (FOV) visual motion stimulus impacted the reliability of a cognitive state ML algorithm developed previously without inclusion of visual motion stimuli in the training data. We used the Visual Vestibular Balance Device (VVBD), a multi-axis human rotation stimulus device with a motorized twelve-foot diameter rigid spherical visual surround to provide an intermittent Earthvertical yaw visual motion stimulus (30deg/sec, $\sim 1 \mathrm{~m} /$ sec tangential) to standing participants during simulated flight. Participants were instrumented with sensors for video-oculography (VOG), heart rate variability (HRV), electroencephalography (EEG), and galvanic skin response (GSR) that input to the ML algorithm. In addition, head accelerometry and stabilometry were recorded during each 40-50-minute simulated cross country flight, along with flight cross track error, time and subjective workload scores. The simulation, presented on a vertically oriented display subtending $\sim 35 \mathrm{x} 60 \mathrm{deg}$, presented four airborne threat warnings that required 
an evasive turn followed by a return to waypoint navigation. The interior illumination of the rotating surround, which has a white background on the internal surface covered with approximately 300 black dots distributed in a hyperuniform pattern, remained off. The interior was illuminated only for the $\sim 3-4$ minutes of each evasive maneuver, twice in the direction of the turn and twice in the opposite direction. A secondary working memory "N-back" task embedded in the instrument panel ran continuously $(\mathrm{N}=2)$ throughout the flight. The VVBD internal participant restraint system allows postural sway on the stabilometry platform and was adjusted vertically to place the participant's head at the center of the sphere. This provides a purely angular motion of the FOV which provides a compelling visually induced motion sensation (VIMS) that must be actively suppressed, increasing cognitive workload and stress while degrading flight and secondary task performance.

\section{Vestibular contribution to balance control during stance}

\section{(Abstract 4)}

Robert J. Peterka, Ph.D.

National Center for Rehabilitative Auditory

Research, VA Portland Health Care System and

Oregon Health \& Science University, Portland,

Oregon

Understanding the role of the vestibular system in balance control requires an appreciation of the fact that balance control is organized as a closed-loop feedback control system and that orientation information from other sensory systems, chiefly proprioception and vision, make major contributions. Each sensory system has limitations. Proprioception, encoding joint kinematics, can only detect body orientation relative to the contact surface. Similarly, vision detects head, and indirectly, body orientation relative to the visual environment. But the contact surface may not be stable and the visual environment may move. Only the inertial signals from the vestibular sensors provide information the brain can use to derive orientation-in-space information. But vestibular orientation information is compromised by its poor signal-to-noise qualities since body motions during stance are small relative to the wide dynamic range of vestibular sensors (van der Kooij
\& Peterka, 2011). The brain's balance control solution is to utilize all available sensory orientation information via a 'sensory integration' mechanism that is able to rapidly adjust the relative contributions of the various sensory systems as environmental conditions change (Assländer \& Peterka, 2016). Experiments that perturb balance using pseudorandom tilts of the stance surface or visual scene evoke body sway can be used to characterize balance control dynamics, and a feedback control model of the balance system, representing sensory integration as a weighted combination of sensory cues, can account for the experimental data (Peterka, 2002). Based on this control model, sensory weights can be estimated and these weights show changes as a function of test conditions (e.g., stimulus amplitude), pathological states (e.g., bilateral and unilateral vestibular loss (Peterka et al. 2018)), and aging. In addition to sensory re-weighting, the balance control system shows a remarkable ability to recalibrate to adjust to altered conditions. As an extreme example, when a robot manipulator was used to reverse the normal relationship between vestibular-derived orientation information and the direction of corrective ankle toque needed for balance, after only $30 \mathrm{~s}$ of training most subjects were able learn this reversed relationship and maintain standing balance (Forbes et al., 2016).

\section{References}

1. Assländer \& Peterka (2016). J Neurophysiol 116:272-285.

2. Forbes et al. (2016). J Neurosci 36:11510-11517.

3. Peterka (2002). J Neurophysiol 88:1097-1118.

4. Peterka et al. (2018). Frontiers Neurology 9:1045.

5. van der Kooij \& Peterka (2011). J Comput Neurosci 30:759-778.

Acknowledgements: NASA NAG5-7869, NIH NIA AG17960, NIDCD DC04082 and DC010779, US Veterans Affairs RR\&D I01RX001951.

\section{Brainstem control of movement initiation for balance}

\section{(Abstract 5)}

David E. Ehrlich 1 Wenze Li 2, Venkata Voleti 2, Elizabeth Hillman 2, David Schoppik 1

1 Neuroscience Institute, Dept. of Otolaryngology, NYU School of Medicine, NY, NY, 10016; 2 
Laboratory for Functional Optical Imaging, Departments of Biomedical Engineering and Radiology, Columbia University, NY, NY, 10027

Animals sense when balance is lost and react with stabilizing movements. To understand how the nervous system transforms sensations of instability into movement, we leveraged the optical accessibility and biomechanical simplicity of larval zebrafish. We previously reported that larvae balance by initiating corrective swimming when pitched nose-up/down from horizontal. To understand how information about head orientation is transformed into a command to initiate movement, we utilized peripheral and central lesions of the vestibular system, and developed a zebrafish application of SCAPE (swept confocally-aligned planar excitation) microscopy for high-speed, volumetric calcium imaging of vestibular responses. We report that genetic loss of pitch-sensitive utricles in otogelin mutants specifically impaired the ability to initiate swims in response to nose-up/down tilts while freely swimming. Calcium imaging revealed that nose-up/ down posture is bilaterally encoded by a population of genetically-defined neurons in the tangential vestibular nucleus. When we lesioned this tangential nucleus population using multiphoton laser ablation or the photosensitizer, killerRed, we found that larvae behaved as though they lacked utricles, failing to initiate swims based on posture. Tangential neurons innervate neurons in the nucleus of the MLF, which directly project to the spinal cord and bilaterally responded to nose-up/down tilts. Similarly to tangential nucleus lesions, laser ablations of the MLF impaired swim initiation based on posture. We conclude that an ascending, brainstem intrinsic pathway is critical for triggering stabilizing reactions when pitch-axis stability is lost.

\section{Frequency analyses of posturography using logarithmic translation}

\section{$\underline{\text { (Abstract 6) }}$}

Takeshi Tsutsumi, MD.; Ph.D.

Ayame Taniguchi, MD.

Takamori Takeda, MD.

Taku Ito, MD.; Ph.D.

Yoshiyuki Kawashima, MD.; Ph.D.

Department of Otorhinolaryngology, Tokyo Medical and Dental University, Tokyo, Japan
Posturography can directly evaluate stability of our body. Frequency analysis of posturography is often adopted, which may significantly contribute to quantitative analysis of stability. However, powerspectral expression of frequency analysis shows a fitting curve with exponential approximation, which is difficult to provide visual information of the relevant frequencies. Whereas, logarithmic translation of exponential approximation formula of power-spectral data $(\mathrm{Y}=\mathrm{aXb})$ can enable linear approximation $(\log \mathrm{Y}=\log \mathrm{a}+\mathrm{b} * \log \mathrm{X})$, which enables us to recognize frequency characteristics as a serviceable information. In this study, we adopted frequency analyses for posturography of healthy subjects and patients with spinocerebellar degeneration (SCD) using logarithmic translation, in order to determine its clinical use in managing the aging population and patients with disequilibrium. 172 healthy subjects and 73 patients with SCD were enrolled in this study. Posturography was performed with their eyes fixed and closed, with and without foam rubber. The power-spectral data, operating with maximum entropy method, were obtained under each direction of antero-posterior and lateral. Power-spectral data were logarithmically translated for quantitative evaluation.

Aging demonstrates the change in characteristics of frequency distribution. Findings from teenagers revealed high-frequency dominant fluctuation. This can be interpreted as proprioceptive compensation for immature postural control in younger age group. On the other hand, in elderly populations, increased frequency characterized by 3 peaks can indicate postural disturbances attributed to each sensory input, possibly $0.15 \mathrm{~Hz}$ for visual, $1.2 \mathrm{~Hz}$ for vestibular, and $4 \mathrm{~Hz}$ for proprioceptive. Disappearance of $1.2 \mathrm{~Hz}$ peak in antero-posterior fluctuation also supports vestibular contribution. The load of foam rubber also removes this peak, which supports this theory.

Visual disturbance (closed-eye condition) enhanced fluctuation around 0.15 and $1.2 \mathrm{~Hz}$, which indicates loss of visual control and consequent vestibular compensation. The peak frequency tends to shift higher in elderly population, possibly representing proprioceptive compensation.

Difference in power-spectral distribution between healthy subjects and patients with SCD demonstrated 2-peaked increase around 0.5 and $2.5 \mathrm{~Hz}$. It seems to have different characteristics from that of the aging. Distribution of power-spectral data could provide disease-specific characteristics, which could be a novel parameter for differential diagnosis. 


\section{A novel ground-based analog of spaceflight neurovestibular stimulation produces sensorimotor impairment}

\author{
(Abstract 7) \\ Jordan B. Dixon, MS, Victoria Brazell, and Torin \\ K. Clark, Ph.D. \\ University of Colorado - Boulder, Boulder, $\mathrm{CO}$
}

Extended exposure to microgravity alters central processing of spatial orientation cues, leading to impaired balance and locomotion, disorientation, and motion sickness when astronauts return to Earth. With relatively few astronauts and challenges accessing the crew, it has remained difficult to quantify impairment immediately post-landing (1), rigorously assess countermeasures (2), and develop a well-support conceptual understanding of neurovestibular adaptations to spaceflight (3). To address this, we have proposed a novel paradigm to partially replicate the neurovestibular stimulation experienced during spaceflight. In this analog, termed the wheelchair head immobilization paradigm (WHIP), the subject lies on their side with their head restrained in a custom facemask, aligning their interaural axis with gravity. The subject's bed is attached to a modified electric wheelchair which the subject drives using a joystick. WHIP alters the vestibular stimulation in a single axis (pitch), similar to microgravity exposure, by preventing coupled rotation and tilt, removing the tonic loading of gravity from the sagittal plane, while still enabling pitch rotation and naso-occipital translation. We hypothesize extended exposure to WHIP will cause central reinterpretation of neurovestibular cues, leading to sensorimotor impairment post-WHIP, similar to that observed post-spaceflight. To assess this, for $\sim 12$ hours we exposed nine WHIP subjects and two control subjects who remained on their sides and drove the wheelchair bed but were not head-restrained. Before and after exposure, subjects reported perceived orientation/motion and performed functional sensorimotor tests, similar to those assessed in astronauts. All paradigm subjects systematically reported illusory perceptions of selfmotion post-WHIP, analogous to returning astronauts (4). Performance on a 15 second standing balance task (on medium-density foam with eyes closed while making $+/-20^{\circ}$ pitch head tilts) significantly deteriorated, from an average of $7 / 8$ successes pre-
WHIP to $4.6 / 8$ post-WHIP ( $\mathrm{p}=0.0087$ ). Further, in a heel-to-toe tandem gait task, the number of correct steps significantly decreased post WHIP $(\mathrm{p}=0.028)$. The control subjects neither appeared to have sensorimotor decrements nor reported similar illusions, suggesting the effects of WHIP were primarily due to the altered neurovestibular stimulation. Future work aims to better quantify the mechanism(s) of impairment and assess WHIP as a testbed for sensorimotor countermeasure design.

\section{References}

1. Tomliovskaya et al (2014) NASA HRP Investigator's Workshop.

2. Bloomberg et al (2016) NASA HRP Sensorimotor Evidence Report.

3. Merfeld (2003) Journal of Vestibular Research 13(4-6).

4. Reschke and Clément (2018) Acta Astronautica 152.

\section{Removing vision reduces head stabilization during dynamic tasks in individuals with unilateral vestibular hypofunction}

\section{(Abstract 8)}

\author{
*Brian J Loyd, PT, PhD \\ University of Utah Physical Therapy, Salt Lake \\ City, UT \\ *Janie Savier-Steiger, BS \\ University of Utah Physical Therapy, Salt Lake \\ City, UT \\ *Serene Paul, PhD, BAppSc(Phty)(Hons) \\ University of Sydney, Sydney Australia \\ * Mark Lester, PT, PhD \\ Army Baylor Physical Therapy, San Antonio, TX \\ *Peter Fino, PhD \\ University of Utah Health Kinesiology and \\ Recreation, Salt Lake City, UT \\ *Lee Dibble, PT, PhD \\ University of Utah Physical Therapy, Salt Lake \\ City, UT
}

Head stabilization during gait is essential for processing sensory information and for maintaining gaze and postural stability. With vestibular impairment, individuals may not effectively stabilize their head, contributing to gait instability, motion 
sensitivity, and increased risk for falls. The purpose of this study was to characterize head stabilization during two sensory conditions in people with vestibular schwannoma resection (PwVSR) and healthy controls (HC). Fourteen PwVSR ( 6 weeks post-surgery) and $15 \mathrm{HC}$ were included. Participants performed items 1 and 8 from the Functional Gait Assessment (FGA) (self-selected gait speed with eyes open and self-selected gait speed with eyes closed, respectively). Linear acceleration was recorded by an inertial sensor (Opal, APDM inc.) worn on the head, and quantified using root mean square (RMS) analysis. RMS values in the anteriorposterior (AP), medial-lateral (ML), and cranialcaudal (CC) planes were calculated for walking periods and were normalized to gait speed. Linear mixed models were fit for each RMS value and adjusted for group, condition, and group by condition interaction. Post-hoc contrasts were run, adjusting for multiple comparisons ( $\mathrm{p} 0.013$ ). A significant group effect was found, with PwVSR on average having significantly greater RMS values in all three planes than HC. Additionally, the group by condition interaction showed that RMS change from vision present to no vision was significantly higher in PwVSR than HC in all three planes. After 6 weeks of recovery, PwVSR appear to adapt to vestibular loss in part by using visual information to stabilize their head during gait. The removal of vision during gait resulted in significant increases in head accelerations in all planes in PwVSR suggesting impairment in the sensory integration processes for effective head stabilization. Future research should determine the persistence of head stabilization deficits in PwVSR beyond 6 weeks and if head acceleration metrics respond to vestibular rehabilitation.

\section{References}

1. Paul et al. 2017 IEEE. 25 (12)

2. Paul et al. 2018 Neural Rehabilitation and Neural Repair. 32 (4)

Acknowledgments: Funded by the U.S. Army Advanced Medical Technology Initiative Grant.

\section{One-year retention of VOR gain, posture and gait after incremental VOR adaptation training in severe bilateral vestibular hypofunction: A case study}

\section{(Abstract 9, Poster Location M)}

\author{
Michael C. Schubert PT PhD \\ Johns Hopkins University, Baltimore MD
}

Yoav Gimmon PT PhD

Faculty of Health Sciences, Ben-Gurion University of the Negev, Beer-Sheva Israel and SpotOn Therapeutics Ltd, Tel-Aviv, Israel

Kyoung Jae Kim $\mathrm{PhD}$

University of Miami, Coral Gables FL

Americo A. Migliaccio PhD

Neurological Institute of Australia, Sydney Australia

A novel training method known as incremental VOR adaptation (IVA) can improve the vestibulo-ocular reflex (VOR) gain for both active and passive head rotation by coupling active head rotations with a laser-projected target that moves in the opposite direction of the head at a fraction of the head velocity. A 51-year-old male with bilateral vestibular hypofunction participated in a research protocol using a portable IVA device for 645 days. After 1 week of training, the passive VOR gain increased greater than $600 \%$ for rightward and $92 \%$ for leftward head rotations. After 1 year of home training, the patient's rightward passive VOR gain reduced a bit but was still elevated by $380 \%$; leftward passive VOR gain only reduced by $6 \%$. While walking, mediolateral (ML) upper body sway decreased $35 \%$ at the end of the training week and after 1 year of training was still improved by $31 \%$. Postural sway area reduced $292 \%$ at the end of week 1; 1 year later the sway area was still reduced by $160 \%$. This case study demonstrate functionally relevant improvement is possible with IVA training after chronic, severe bilateral vestibular hypofunction. Our data suggest retention of motor learning within the vestibulo-ocular and vestibulospinal systems using the IVA method. A clinical trial is underway to compare IVA vs the traditional method of vestibular rehabilitation for vestibular hypofunction. 


\section{Vestibular physical therapy in individuals with cognitive impairment: a theoretical framework}

\section{(Abstract 10, Poster Location K)}

Brooke N. Klatt, PT, PhD

Johns Hopkins University, Baltimore MD

Julie D. Ries, PT, PhD

Marymount University, Arlington VA

Pamela M. Dunlap, PT

University of Pittsburgh, Pittsburgh PA

Susan L. Whitney, PT, PhD, ATC, FAPTA

University of Pittsburgh, Pittsburgh PA

Yuri Agrawal, MD, MPH, FACS

Johns Hopkins University, Baltimore MD

Falls are a major healthcare concern in individuals with Alzheimer Disease (AD) and their caregivers. Vestibular impairment is a known risk factor for falls, and individuals with AD have been shown to have an increased prevalence of vestibular loss compared to age-matched controls. Vestibular physical therapy (VPT) is effective in improving balance and reducing fall risk in cognitively-intact persons with vestibular impairment. However, the effectiveness of VPT in improving balance and reducing falls in individuals with $\mathrm{AD}$ who have vestibular loss has never been explored. In this project, we apply prevailing ideas about rehabilitation and motor learning in individuals with cognitive impairment (IwCI) to VPT. We propose a modification of current evidence-based VPT protocols for IwCI using the Strength-Based theoretical framework that emphasizes the motor learning abilities of IwCI. Additionally, we highlight the importance of establishing an excellent rapport with IwCI, and present key strategies for optimizing the therapeutic relationship. This theoretical framework, which can be applied to clinical practice and clinical research trials, has been accepted for publication in the Journal of Neurologic Physical Therapy for March 2019 publication. In ongoing work, we are assessing the efficacy of this modified VPT protocol in IwCI to improve balance and reduce falls and at the time of presentation we are hopeful that we will have some preliminary results to share.

\section{References}

1. Harun A, Oh ES, Bigelow RT, Studenski S, Agrawal Y. Vestibular Impairment in Dementia. Otology \& Neurotology. 2016;37(8):1137-1142.

2. Hall CD, Herdman SJ, Whitney SL, et al. Vestibular Rehabilitation for Peripheral Vestibular Hypofunction: An Evidence-Based Clinical Practice Guideline: FROM THE AMERICAN PHYSICAL THERAPY ASSOCIATION NEUROLOGY SECTION. Journal of neurologic physical therapy : JNPT. 2016;40(2):124-155.

3. Ries J. Rehabilitation for Individuals with Dementia: Facilitating Success. Current Geriatrics Reports. 2018

Acknowledgements: Funded by NIA (\#RO1 AG057667), NIH/NIDCD (\#R03 DC015583), and NIH/NIDCD (\#K23 DC013056).

\section{The use of tablet technology for objective exercise monitoring in vestibular rehabilitation}

\section{$\underline{\text { (Abstract 11) }}$}

Hovareshti P, Holt L, Gao P, Xiao L, Tolani D, Whitney SL.

Determining vestibulo-ocular reflex (VORx1) exercise compliance for clients with central or peripheral vestibular disorders is challenging for rehab professionals. In addition, providing timely individualized feedback is not feasible since clients perform most of the exercises outside the physical therapy clinic. Here we describe an innovative system for home use to objectively track exercise compliance and provide feedback to both the treating physical therapist and the client. The system is implemented as a tablet-based app. Video instructions help clients recall how to do the VOR exercises and a metronome guides head speed during the exercises. The system collects symptom data before and after each exercise which is then combined with objective performance data to provide feedback to the client and the physical therapist. A gamified reward system is used to motivate clients and improve exercise compliance. With the tablet camera, facial and eye recognition is used to analyze accuracy of the head and eye movement during the exercise. Convolutional 
Neural Network (CNN) models are used to determine the face image, refine landmark points (eye and nose positions), and capture face shape. Head angles are computed using the filtered data from facial landmarks. A peak detection algorithm is used to determine head-speed compliance. A CNN trained to classify eye gaze direction as on- and off-target is then used to determine eye-gaze compliance. Publically available data from 15 persons was utilized to train and evaluate the eye-gaze model, yielding $96 \%$ accuracy using 5 -fold cross validation. All of these data are stored in a cloud to support remote monitoring by the rehab provider. By monitoring exercise performance and enabling objective feedback, this innovative system can greatly improve exercise compliance outside the clinical environment. With access to detailed performance data, physical therapists can better manage client progression between visits, thus optimizing vestibular rehabilitation outcomes.

This work is supported by the US Army Medical Research and Materiel Command under Contract Number listed on W81XWH-18-C-0030. The views, opinions and/or findings contained in this report are those of the author(s) and should not be construed as an official Department of the Army position, policy or decision unless so designated by other documentation.

\section{First-in-human safety and preliminary efficacy results for the labyrinth devices MVI vestibular implant}

\section{(Abstract 12)}

Peter J Boutros ${ }^{1}$, Mehdi Rahman ${ }^{1,2}$, Nicolas S Valentin $^{1,2}$, Desi Schoo1, Margaret Chow ${ }^{1}$, Andrianna Ayiotis ${ }^{1}$, Brian Morris ${ }^{1}$, Kelly Lane ${ }^{1}$, Stephen Bowditch ${ }^{1}$, Yoav Gimmon ${ }^{1}$, Andreas Hofner $^{3}$, Aitor Morillo Rascon ${ }^{3}$, Andreas Marx ${ }^{3}$, Ross Deas ${ }^{3}$, Stefan Strasser ${ }^{3}$, Gene Y Fridman1, Natan S Davidovics ${ }^{1,2}$, Dale C Roberts ${ }^{1}$,

Abderrahmane Hedjoudje ${ }^{1}$, Bryan K Ward ${ }^{1}$, Carolina Treviño $^{1}$, Michael Schubert ${ }^{1}$, John P Carey ${ }^{1}$, Andreas Jaeger $^{3}$, Charles C Della Santina ${ }^{1,2,3 *}$

${ }^{1}$ Vestibular NeuroEngineering Laboratory, Johns

Hopkins School of Medicine, USA;

${ }^{2}$ Labyrinth Devices, LLC, USA;

${ }^{3}$ Med-El GmbH, Austria; *presenter
Purpose: In rodents and nonhuman primates with bilateral vestibular hypofunction, we previously demonstrated that motion-modulated current pulses delivered via electrodes in the ampullae can partially restore the 3D angular vestibulo-ocular reflex (VOR) while partially preserving hearing. Based on those studies, we initiated a first-in-human clinical trial of continuous motion-modulated prosthetic stimulation using the MVI Multichannel Vestibular Implant System $^{\mathrm{TM}}$ developed by Labyrinth Devices LLC and MedEl GmbH.

Methods: Six subjects underwent unilateral implantation; two were observed overnight and four were discharged the same day. VOR responses to 0.5-5 Hz passive head or whole-body rotation and prosthetic stimulation; pure tone and speech audiometry; and metrics of posture, gait, dynamic visual acuity and quality of life were assessed during 6-30 months of continuous MVITM use.

Results: Electrically-evoked VOR responses typically ranged from $5-40 \%$ and aligned approximately with the stimulated canal for at least one electrode in each of three canals in each implanted ear. VOR responses were typically smaller than those for similar currents in animals, but significantly greater with motion-modulated stimulation than without. Motion perception thresholds are typically below VOR thresholds. Hearing sufficient for unaided communication was preserved in 5 of 6 subjects. Objective posture and gait metrics reveal a trend toward improvement relative to preop and significantly improved performance with motionmodulated input versus placebo stimulation.

Conclusions: The MVI ${ }^{\mathrm{TM}}$ system can be implanted in outpatient surgery, generates motion percepts and 3D binocular VOR responses consistent with selective stimulation of 3 implanted canals, can be implanted with preservation of useful hearing and provides sensory information that facilitates posture and gait.

Disclosures: This research was supported by NIH/ NIDCD R01DC013536, R01DC9255 and T32DC000023, performed under Johns Hopkins School of Medicine IRB protocol NA00051349 and US FDA IDE G150198, and registered at ClinicalTrials.gov \#NCT02725463. CCDS holds an equity interest in Labyrinth Devices LLC; terms of this arrangement are managed in accordance with university policies. 


\section{Using vestibular prosthesis to restore yaw rotation perception in primates with bilateral vestibular hypofunction}

\author{
(Abstract 13, Poster Location AA)
}

Kantapon Wiboonsaksakul ${ }^{1}$, Charles C Della Santina $^{2}$, Kathleen E Cullen ${ }^{1}$

${ }^{1}$ Department of Biomedical Engineering, Johns

Hopkins University

${ }^{2}$ Department of Otolaryngology-Head and

Neck Surgery, Johns Hopkins University

School of Medicine

The vestibular system senses movement of the head and provides information crucial for appropriate reflexes and vestibular perception. Patients with bilateral loss of vestibular function not only experience disequilibrium, visual instability, and dizziness due to impaired vestibular reflexes but also suffer from loss of vestibular perception. To help improve the quality of life of these patients, there has been a recent focus on the development of devices to replace vestibular function. One emerging approach has been centered on a prosthesis that senses head rotation and translates the movement into ampullary stimulation, substituting for the damaged vestibular periphery. Recent experiments in our groups have characterized the efficacy of our vestibular prosthesis on the vestibulo-ocular reflex (VOR) and vestibulocollic reflex in nonhuman primates. However, to date, its ability to restore vestibular perception in nonhuman primates has not been explored. Here, we investigated the use of the vestibular prosthesis to restore yaw rotation perception in primates. A unilaterally vestibulardeficient rhesus monkey was trained to distinguish between passive left and right yaw rotations in a two alternative forced-choice recognition task. After the performance stabilized, the monkey underwent prosthetic implantation on the normal side, resulting in a bilateral vestibular hypofunction (VOR gain < 0.15 bilaterally). We quantified the task performance and generated psychometric curves in light (with visual cues) and in darkness (without visual cues) for presurgery (unilaterally deficient), post-surgery (bilaterally deficient), and prosthesis (bilaterally deficient with prosthesis on) conditions. Prior to the surgery, the supra-threshold $(30 \mathrm{deg} / \mathrm{s}$ at $1 \mathrm{~Hz}$ ) performance in darkness was stable at $\sim 75 \%$, and dropped to chance $(\sim 50 \%)$ after the implantation.
Using the standard stimulation mapping, sessions with the prosthesis on showed a significant increase $(\mathrm{p}<0.05)$ in performance to $\sim 60 \%$, indicating an improvement in yaw rotation perception. Prosthesison sessions also showed a significant increase $(\mathrm{p}<$ 0.001 ) in response preference toward the ipsilateral side, likely due to the incomplete adaptation to baseline stimulation. Even with the partial adaptation, a profound rebound preference toward the contralateral side was observed immediately after the prosthesis was turned off. Future work will investigate the neural correlates of the perceptual improvement, in addition to employing a comparable psychophysical-based methodology in human patients.

\section{Recovery of head-trunk kinematics during functional movement tasks following unilateral vestibular differentiation}

\section{(Abstract 14)}

Lee Dibble, PhD, PT

Brian Loyd, PhD, PT

Grace Hunt, BS

University of Utah Department of Physical Therapy and Athletic Training

Richard Gurgel, MD

Clough Shelton, MD

University of Utah Department of Surgery, Division of Otolaryngology, Salt Lake City, UT

Mark Lester, PhD PT

Army-Baylor University, Fort Sam Houston

San Antonio, TX

Serene Paul, PhD, PT

University of Sydney, Discipline of Physiotherapy

Sydney, NSW, Australia

Head accelerations during functional movement tasks (sit to stand and 180 degree turns) and head stabilization during repetitive dynamic movement (straight walking) may be particularly challenging for individuals with acute unilateral vestibular deafferentation. The purpose of this study was to utilize functional mobility tasks that required head angular accelerations to examine head and trunk kinematics longitudinally and compare these 
behaviors to individuals without vestibular hypofunction. 12 participants post vestibular schwannoma resection (VSR) and 20 individuals without vestibular hypofunction. Time to perform TUG task and gait speed were measured with a stop watch. Pitch plane head and trunk amplitude (deg) and velocity (deg/se) during sit-to-stand (STS); yaw plane head and trunk velocity $(\mathrm{deg} / \mathrm{sec})$ during the first turn of the TUG; Head and trunk yaw plane amplitude (deg), head and trunk peak yaw plane angular velocity (deg) during gait. Data were gathered using wearable inertial measurement units. Differences in outcomes between individuals with VSR at 4 days compared to 6 weeks post-surgery were examined using Wilcoxen signed rank tests. Comparison in yaw plane kinematics between participants with VSR and controls were examined with Mann Whitney U tests. From acute to sub-acute time periods, participants post VSR demonstrated significant improvements in yaw and pitch plane angular velocity ( $p$ 0.003). Yaw plane velocity during the TUG turn remained significantly reduced compared to participants without VSR (p 0.003). Head motions during straight ahead gait were not responsive to within subject recovery or crosssectional differences to controls. After six weeks of recovery with their new asymmetrical vestibular afference, individuals experienced substantial recovery in head and trunk movement kinematics. However, this recovery appears incomplete especially when considering head and trunk yaw plane movement velocity during 180 degree turns in the TUG task.

\section{References}

1. Mijovic T, et al Otol Neurotol. 2014;35(10):e348357.

2. Borel L, et al Brain. 2002;125(pt 4):880-894.

Paul SS, et al JAMA Otolaryngol Head Neck Surg. 2017;143(10):1008-1014.

Acknowledgments: This study was funded by a U.S. Army Advanced Medical Technology Initiative Grant 2015-2016

\section{Gait changes during dual-task walking in individuals with and without visual vertigo}

\section{$\underline{\text { (Abstract 15) }}$}

Pamela M. Dunlap, DPT*

Ervin Sejdic, PhD, Associate Professor*

Carrie W. Hoppes, PT, PhD, Assistant Professor, Army-Baylor, Fort Sam Houston, TX

Kefah M. Alshebber, MSPT, PhD*

Brooke N. Klatt, DPT, PhD, Postdoctoral Research Fellow, Johns Hopkins University, Baltimore, MD

Colleen Danaher, DPT, Mandell Center for

Multiple Sclerosis, Saint Mary's Hospital,

Waterbury, CT

Andrea L. Rosso, PhD, MPH, Assistant Professor*

Joseph M. Furman, MD, PhD, Professor*

Susan L. Whitney, DPT, PhD, Professor*

*University of Pittsburgh, Pittsburgh, PA

Persons with a vestibular disorder walk more slowly when confronted with a simultaneous cognitive task. The presence of visual vertigo (VV) may further impair walking in persons with a vestibular disorder. The purpose of this research was to examine gait changes under different surface and task conditions among patients with vestibular disorders who also manifested VV.

Fourteen subjects with VV and fourteen age and gender-matched control subjects (CON) were included. A cross-sectional experimental study with four combinations of two surfaces (even and uneven) and two task conditions (single and dual-task) was performed. The single task consisted of even walking (EW) or uneven walking (UW), and the dual-task consisted of reciting every other letter of the alphabet aloud while walking (EWABC or UWABC). Accelerometers were worn at the lumbar spine at the level of the iliac crests. Mixed ANOVA were conducted to determine the differences among gait speed, standard deviation of the signal amplitude (SD) in the mediolateral (ML) direction, and the peak frequency (PF) in the anteroposterior (AP) direction at the trunk across trials, conditions, and between groups.

There were no differences in age $(\mathrm{M}=39 \mathrm{y}$ [11]), height, weight, or education level between the two groups. Overall, the VV group walked more slowly than the CON group $\left(\mathrm{F}=12, \mathrm{p}=.002, \eta^{\wedge} 2=.325\right)$. Both groups walked more slowly when encountering 
an uneven surface or when posed with a dual task activity $\left(F=131, p=.001, \eta^{\wedge} 2=.84\right)$. For SD in the ML direction, differences among conditions $[\mathrm{F}=24$, p.001, $\eta^{\wedge} 2=.49$ ) indicated greater ML variability in the UW condition and less ML variability in EWABC condition compared to EW and UWABC. The CON group had greater PF in the AP direction than the VV group $\left[F=5.8, p=.024, \eta^{\wedge} 2=.189\right]$. There were no differences between groups in the number of alphabet sets completed or mistakes made on the cognitive task.

Our findings indicate both persons with VV and healthy adults slow walking speed and demonstrate greater ML variability when confronted with uneven terrain and cognitive tasks. Persons with VV exhibit significantly slower gait speed than health adults under all conditions, which may have implications for potential fall risk and the development of interventions.

\section{References}

1. Roberts, (2011); Nascimbeni, (2010); Sejdic, (2014)

Acknowledgements: Supported by (P30AG024827) and (UL1TR001857).

\section{Patient attitudes toward vestibular disorders}

\section{(Abstract 16)}

Whitney Chiao, BA ${ }^{1}$; Roseanne Krauter ${ }^{1}$, MSN, FNP-C; Laura Kirk ${ }^{1}$, PA-C; Kristen K. Steenerson², MD; Jeffrey Sharon ${ }^{1}$, MD

${ }^{1}$ University of California, San Francisco

${ }^{2}$ Stanford University

Introduction: Dizziness and vertigo are common complaints. Although there are some qualitative studies interviewing patients, patients' attitudes regarding their dizziness, provider capabilities, and receptiveness toward therapy have not been formally studied.

Methodology: This was a cross-sectional study. The main outcome measure was a non-validated questionnaire regarding patients' beliefs regarding the cause of their dizziness, likelihood of successful treatment, and openness to treatment.
Results: 67 surveys were completed between January 2017 and September 2018. A majority of patients attributed their dizziness to their ears $(n=47$, $70 \%)$, followed by the brain $(n=29,43 \%)$. Most subjects chose "neither agree nor disagree" about whether their provider could identify the cause of their dizziness $(27 \%)$. Most subjects also chose "neither agree nor disagree" that their dizziness would resolve with treatment (31\%). These attitudes were not influenced by demographics, dizziness severity, anxiety, depression, or quality of life on multivariate ordinal regression modelling.

Conclusion: A plurality of patients with dizziness have neutral attitudes with regards to believing that their provider can identify the cause of their dizziness and whether their dizziness will resolve with treatment. These attitudes do not differ by demographic information, dizziness handicap, quality of life, depression, or anxiety.

\section{References}

1. Neuhauser HK. The epidemiology of dizziness and vertigo. Handb Clin Neurol. 2016;137:67-82.

2. Kruschinski C, Theile G, Dreier SD, HummersPradier E. The priorities of elderly patients suffering from dizziness: a qualitative study. Eur J Gen Pract. 2010;16(1):6-11.

Acknowledgments/disclosures: None

\section{Acute Sports Related Concussion Affects Central, not Peripheral, Vestibular Function in Collegiate Athletes}

\section{(Abstract 17)}

Graham D. Cochrane, B.A.

Medical Scientist Training Program School of Medicine, University of Alabama at Birmingham

Dr. Jennifer B. Christy, PT Ph.D. and Dr. Anwar Almutairi, DPT Ph.D.

Department of Physical Therapy, University of Alabama at Birmingham

Introduction: Sports-related concussion (SRC) commonly leads to changes in balance, symptoms of dizziness, and nausea with movement. This array of symptoms suggests that the vestibular system may 
be dysfunctional in SRC. However, the exact vestibular deficits caused by SRC are unknown. Understanding the exact mechanisms of damage to the vestibular system and which clinical measures of vestibular function are the most reliable to change in SRC could help suggest and target new interventions, drastically improve patient outcomes.

Objective: The objectives of this study were to: 1) evaluate test-retest reliability and normative data on vestibular and balance tests in a cohort of athletes aged 18-24 years with no current diagnosis of SRC; 2) compare athletes with and without SRC on the protocol subtests; 3 ) identify subtests to be included in standardized clinical concussion testing protocols.

Methods: 87 healthy athletes (mean age 20.6 years; $\mathrm{SD}=1.8$ years; $39 \mathrm{~F}, 48 \mathrm{M}$ ) and 28 athletes (mean age 20.7 years $(\mathrm{SD}=1.9) 11 \mathrm{~F}, 17 \mathrm{M})$ with sports related concussion (SRC) were tested using rotary chair, cervical vestibular evoked myogenic potential (c-VEMP) and the Sensory Organization Test (SOT). A subset $(n=43)$ were tested twice to determine testretest reliability. The data were analyzed for reliability using Intraclass Correlation Coefficient (ICC). Comparisons between athletes with and without SRC were made using Mann-Whitney U and t-tests.

Results: ICCs varied widely for all tests ranging from 0.10 to 0.87 , indicating dramatic differences in clinical utility of these tasks, specifically in regard to the benefit of baseline testing common in concussion protocols. There was no significant difference between athletes with and without SRC on tests of peripheral vestibular function (i.e. rotary chair simple VOR tasks, c-VEMP). Athletes with SRC had significantly worse scores than athletes without concussion (p0.05) on VOR cancellation gain, subjective visual vertical and horizontal variance, and all conditions of the SOT.

Conclusion: SRC does not appear to affect medium frequency VOR or saccular function. SRC affected the ability of athletes to use vestibular inputs for perception of vertical and postural control and cancellation of the VOR. Vestibular symptoms in SRC may arise from changes in central processing of vestibular information.

\section{Video ocular counter-roll (vOCR): A new clinical test of otolith-ocular function}

\section{(Abstract 18)}

Francesco Fornasari ${ }^{1}$, Shirin Sadeghpour ${ }^{2}$, Jorge

Otero-Millan², David S Zee ${ }^{3}$, John P Carey ${ }^{4}$, Amir

Kheradmand $^{5}$

${ }^{1}$ San Raffaele University, Italy)

${ }^{2}$ Johns Hopkins, Department of Neurology, USA)

${ }^{3}$ Johns Hopkins, Departments of Neurology, Neuroscience, Ophthalmology, and Otolaryngology \& Head and Neck Surgery, USA)

${ }^{4}$ Johns Hopkins, Department of Otolaryngology \& Head and Neck Surgery, USA)

${ }^{5}$ Johns Hopkins, Departments of Neurology and Otolaryngology \& Head and Neck Surgery, USA)

The video head impulse testing (vHIT) is now widely used for quantification of semicircular canal function. However, a video-oculography (VOG) method for evaluation of otolith-ocular function is lacking. Here, we investigated whether the VOG quantification of ocular counter-roll (vOCR) during static head tilts can be used to detect loss of vestibular function. The feasibility of vOCR testing at the bedside was assessed using simple lateral head-onbody and whole-body tilts of 30 degrees. We recruited patients with vestibular loss from resection of vestibular schwannoma within three weeks (acute), four weeks to six months (subacute), and more than six months (chronic) after their surgeries. The vOCR and vHIT measurements were compared among the patient groups and age matched healthy controls. The average vOCR values from patients were significantly lower compared with the healthy controls during both head-on-body and whole-body tilts ( $p$ values 0.05 ). In the acute group, vOCR was reduced on the side of vestibular loss compared to the healthy side ( $p$ values 0.05 ). This asymmetry was more pronounced for the whole body tilt than the head-on body tilt in the acute and subacute groups, but it resolved in the chronic group ( $p$ values 0.5 ). The average vHIT gains on the side of vestibular loss were lower than the healthy side in all acute, subacute, and chronic patients ( $\mathrm{p}$ values 0.05 ). These results show that the vOCR can be easily applied at the bedside to detect and track loss of otolith-ocular 
function at different stages of recovery from vestibular loss. In the acute stage, both vOCR and vHIT show an asymmetry with lower gains on the side of vestibular loss. While vHIT asymmetry persists with loss of vestibular function, vOCR asymmetry resolves over time.

\section{Evaluation of gravitational recognition in patients with spinocerebellar degeneration using the listing's plane}

\section{(Abstract 19)}

Takamori Takeda $\mathrm{MD}^{1}$, Takuo Ikeda, $\mathrm{MD}, \mathrm{PhD}^{2}$, and Takeshi Tsutsumi MD, $\mathrm{PhD}^{1}$

${ }^{1}$ Department of Otolaryngology, Tokyo Medical and Dental University, Tokyo, Japan

${ }^{2}$ Department of Otorhinolaryngology, Tsudumigaura medical center for children with disabilities

Purpose: Listing's plane reflects gravitational recognition, constructed through integration of visual, vestibular, and proprioceptive sensory inputs in neural integrator of central nervous system. Spinocerebellar degeneration (SCD) is a progressive degenerative disease mainly involving cerebellum and brainstem, and leads to disturbance of the integration of 3 sensory inputs. Such mis-integration should destabilize the construction of the gravityoriented internal model, resulting in thickening of Listing's plane. Recently, we established a method of calculating the thickness of Listing's plane obtained from 3-dimensional eye movements using videooculography. The purpose of this study is to evaluate the gravitational recognition in patients with $\mathrm{SCD}$, using the thickness of the Listing's plane as a parameter, and investigate the association between other neuro-otological examinations (electronystagmography (ENG) including caloric test and posturography) and thickness of Listing's plane.

Methods: Thirty-two healthy subjects and 21 patients who were diagnosed with SCD by neurologists between 2016 and 2018 were enrolled in this study. The subjects were wearing infrared CCD-mounted goggles in the sitting and supine position, leaving both eyes unobstructed. Fifty $\mathrm{cm}$ in front, we placed a screen on which were 9 fixation points. The subjects were instructed to fixate for $1 \mathrm{~s}$ on each of the 9 fixation points consecutively. We digitized the video recording using a macro program for ImageJ and calculated thickness of the Listing's plane on each SCD patients and healthy subjects.

Results: We identified thickening of Listing's plane in SCD patients only when they kept sitting position. ENG recordings showed that abnormal eye movements, indicating cerebellar deficit, associated with thickening of Listing's plane in response to positional change. The thickness of the Listing's plane correlated neither caloric response nor posturography results.

Conclusion: The thickness of the Listing's plane can provide parameters for evaluation of instability of gravitational recognition in patients with SCD.

\section{Vestibular impairment predicts poorer spatial cognition and driving difficulty in patients with alzheimer's disease}

\section{(Abstract 20, Poster Location N)}

Eric X. Wei*; Esther S. Oh, MD, $\mathrm{PhD}^{\wedge}$; Aisha

Harun, MD*; Yuri Agrawal, MD, MPH*

*Department of Otolaryngology - Head and Neck

Surgery, Johns Hopkins University School of Medicine

^Department of Medicine, Johns Hopkins University School of Medicine

The vestibular system is an important contributor to balance control, spatial orientation, and falls risk. Recent evidence has shown that Alzheimer's disease (AD) patients have a higher prevalence of vestibular dysfunction relative to healthy controls. We sought to evaluate whether vestibular impairment is specifically associated with poor spatial cognitive skills among patients with mild cognitive impairment (MCI) and AD. We enrolled 50 patients (22 MCI and 28 AD) from an interdisciplinary Memory Clinic and measured vestibular physiologic function in all patients. In this study we focused on saccular function measured with the cervical vestibular- 
evoked myogenic potential (cVEMP) given prior work showing an association between saccular function and spatial cognition in healthy adults. Spatial cognitive function was assessed using neurocognitive tests including the Money Road Map Test (MRMT). General cognitive function was assessed with the Mini-Mental Status Examination (MMSE). Additionally, we administered a questionnaire that assessed real-life correlates of spatial impairment, including driving difficulty, losing objects, falls, and fear of falling. Among MCI patients, $9(40.9 \%)$ had unilateral and $5(22.7 \%)$ had bilateral vestibular loss. Among AD patients, 8 (28.6\%) had unilateral and $16(57.2 \%)$ had bilateral vestibular loss. In multiple linear regression analyses adjusted for age, gender, education level, and MMSE, MCI and AD patients with saccular impairment made significantly more errors on the MRMT relative to patients with normal saccular function ( $\beta=7.3$ errors, $p=0.004$ for unilateral and $\beta=6.4$ errors, $p=0.006$ for bilateral saccular impairment). Moreover, in multiple logistic regression analyses, MCI and $\mathrm{AD}$ patients with bilateral saccular impairment had a significant, greater than 12-fold increased odds of driving difficulty (OR 12.1, p = 0.03) compared to MCI and $\mathrm{AD}$ patients with normal saccular function, and the association appeared to be mediated by spatial cognition as measured by the MRMT. These findings support the hypothesis that vestibular loss contributes specifically to a decline in spatial cognitive ability in MCI and AD patients, independently of general cognitive decline, and may predict a "spatially impaired" subtype of AD.

\section{References}

1. Allan et al. (2009). PLoS One 4, e5521.

2. Bigelow et al. (2015). J Am Geriatr Soc 63, 18371844.

3. Harun et al. (2016). Otol Neurotol 37, 1137-1142.

4. Scheltens et al. (2015). J Neurol Neurosurg Psychiatry 87, 235-243.

Acknowledgements: Funded by NIH/NIDCD K23AG043504-01, T32DC000027-25, K23DC013056-02, and P50AG005146-32S1.

\section{Noise-induced vestibular injury and motor impairment}

\section{(Abstract 21, Poster Location U)}

Courtney Stewart*, Ph.D.; David Bauer, BA; Ariane Kanicki, MS; Richard Altschuler, Ph.D.; W. Michael King, Ph.D.

University of Michigan, Department of Otolaryngology Head/Neck Surgery and Kresge Hearing Research Institute, Ann Arbor MI

The vestibular system plays a critical role in detection of head movements and is essential for normal postural control and balance. Because of their anatomical proximity to the cochlea, the otolith organs are vulnerable to sound pressure and at risk for noise-overstimulation. It was previously reported that human subjects with sensorineural hearing loss had abnormal cervical vestibular-evoked myogenic potential (cVEMP) responses and higher thresholds for eliciting cVEMP responses than age-matched controls that did not have sensorineural hearing loss (Akin et al., 2012; Zuniga et al., 2012). We have reported noise-induced reductions in vestibular short latency evoked potential (VsEP) responses in rats with significant loss of calyceal endings in the sacculus (Stewart et al., 2018). Taken together, these data suggest that noise may impair vestibular signaling in descending vestibular inputs, but the functional consequences of this impairment are unclear. It is known, however, that irregularly discharging vestibular afferents are the most soundsensitive (Murofushi \& Curthoys, 1997) and this population of afferents plays an important role in descending vestibular reflexes (Bilotto et al., 1982). The goal of the current study is to correlate changes in VsEP waveform amplitudes and jerk thresholds for evoking VsEPs with changes in rats' ability to cross a narrow balance beam. We hypothesize that noise-induced reductions in vestibular nerve activity will be linked to changes in motor performance in vestibular-dependent tasks. Adult Long-Evans rats $(400-450 \mathrm{~g})$ were exposed to intense noise $(120 \mathrm{~dB}$ SPL, $0.5-4 \mathrm{kHz}$ ) for six hours on a single day. Changes in auditory function were evaluated by measuring auditory brainstem response (ABR) before and after noise exposure. Before and across time points up to 28 days after noise exposure, the VsEP was evaluated. Balance beam crossings were analyzed for overall crossing time, foot slip errors, 
and falls. Additionally, probability density functions of head acceleration were used to identify changes in balance beam crossing strategy. Following noise exposure, the VsEP P1N1 waveform (reflecting vestibular nerve activity) was abolished in response to weak $(<1.1 / \mathrm{ms})$ jerk stimuli, and attenuated in response to larger stimuli. This attenuation persisted for up to four weeks in most animals. Additionally, there was a shift in latency for the onset of the P1 waveform in measurable responses. This shift generally recovered despite a persistent reduction in amplitude. When pooled across all noise exposed animals, balance beam crossing times were not significantly different from baseline, despite significant and persistent increases in balance beam crossing times in some animals. Furthermore, the frequency at which the head moved during crossing and the intensity at which this movement occurred was significantly reduced in animals that had longer crossing times, suggesting increased caution after noise-induced injury to the vestibular periphery. Reduced VsEP amplitudes and mildly impaired motor performance in a balance beam crossing task suggest a subtle vestibular-mediated motor impairment after noise-induced injury to the vestibular periphery. These results highlight a need for the further development of dynamic motor assessment tools to evaluate otolith function after vestibular injury.

\section{References}

1. Akin FW et al. (2012) Ear Hear 33(4).

2. Zuniga MG et al. (2012) Otol Neurotol 33(9).

3. Stewart CE et al. (2018) J Neurophys 119.

4. Murofushi $T$ and Curthoys IS (1997) Acta Otolaryngol 117(1).

5. Bilotto G et al. (1982) Exp Brain Res 47.

Acknowledgements: Funded by NIH/NIDCD T32 DC000011 (HBCS Program 2016-2018), F32 DC017063 (2018-2019), R21 DC015097; and the Department of Veterans Affairs 1I01RX001986.

\section{Acute Vestibular Findings in an acquired neurosensory dysfunction}

\section{(Abstract 22)}

\author{
Michael E. Hoffer, MD ${ }^{1,2}$, Bonnie Levin, $\mathrm{PhD}$ \\ ${ }^{3}$, Hilary Snapp, AuD, $\mathrm{PhD}^{1}$, Jim Buskirk ${ }^{1}$, \\ Carey Balaban, $\mathrm{PhD}^{4,5,6,7}$ \\ ${ }^{1}$ Department of Otolaryngology, University of Miami \\ Miller School of Medicine (Miami, Florida) \\ ${ }^{2}$ Department of Neurological Surgery, University of \\ Miami Miller School of Medicine \\ ${ }^{3}$ Department of Neurology, University of Miami \\ Miller School of Medicine \\ ${ }^{4}$ Department of Otolaryngology, University of \\ Pittsburgh (Pittsburgh, Pennsylvania) \\ ${ }^{5}$ Department of Neurobiology, University of \\ Pittsburgh \\ ${ }^{6}$ Department of Communication Sciences \& \\ Disorders, University of Pittsburgh \\ ${ }^{7}$ Department of Bioengineering, University of \\ Pittsburgh
}

Background: In late fall 2016 diplomatic personnel residing in Havana began to present with symptoms of dizziness, ear pain, and tinnitus. As these symptoms began to appear in more personnel, an investigation revealed that these individuals reported that the symptoms often emerged after perception of very focal noise and/or a pressure wave.

Methods: This is a review that examines the presenting findings of this group of patients. It examines a cohort of patients seen at the University of Miami as well as a group individual examined in Havana.

Results: All of the symptomatic individuals reported some combination of dizziness, hearing loss, difficulty staying focused/slower processing speed, tinnitus, ear pain, and/or headaches. Dizziness (92\%) and cognitive complaints (56\%) were the most common individual symptoms. All of the 25 affected individuals reported either dizziness or cognitive complaints, with $12 / 25$ (48\%) reporting both symptoms. All 25 individuals had at least one objective test abnormality.

Conclusion: This is the only study of this population during their acute injury phase.. The preponderance 
of evidence suggests that symptoms and signs emerge after perception of a localized loud noise or pressure field. The objective findings reported for the first time here are very similar to findings in mTBI from other sources although some unique features of this exposure pattern.

\section{Synaptopathic correlates of peripheral vestibular hypofunction}

\section{(Abstract 23)}

\author{
L Hoffman, J Saldate, T Prins, F Schweizer, and M \\ Paulin \\ Departments of Head \& Neck Surgery and \\ Neurobiology, Geffen School of Medicine at UCLA; \\ Department of Zoology, University of Otago, \\ Dunedin, New Zealand
}

Peripheral vestibular hypofunction represents a condition borne out of several etiologies, including age, autoimmune disorders, or ototoxicity. It may manifest in various behavioral compromises, but the common element is diminished capabilities of labyrinthine neuroepithelia for head movement coding. Our laboratory has been exploring animal models of vestibular hypofunction through which the cellular and neurophysiologic bases of the coding deficits can be investigated. One such model is the otoferlin-null mouse, exhibiting the genetic deletion of a synaptic protein critical for stimulus-induced exocytosis. Another model involves ototoxicity induced by direct intraperilymphatic administration of a chemotoxin, primarily gentamicin, through which graded hypofunction can be induced. Both models offer avenues to explore different cellular attributes of hypofunction, but at the same time provide critical insight into contributions to normal vestibular function. We have previously shown that exposure to microgravity, in which gravitational loading of the utricle becomes dramatically attenuated, results in a striking decrease in extrastriolar hair cell synapse density (Sultemeier et al. 2017). These data imply the existence of a cellular repertoire that includes synaptic plasticity resulting from diminished inputs to vestibular epithelia. Furthermore, these findings suggest that synaptic alterations may result, either directly or indirectly, from other conditions involving pathogenic etiologies. For example, we have surveyed both the crista and utricle neuroepithelia and found a postsynaptic correlate to the diminished synaptic function in type I hair cells in the otoferlin-null mouse, confirming that the deficit penetrates to virtually all type I hair cells. We are exploring the distribution of synaptic complexes in these epithelia to determine whether deletion of this critical component of the stimulus coupling cascade (i.e. representing a direct synaptopathic condition) influences synapse distributions in type I hair cells. We have previously shown that low-doses of gentamicin, delivered directly to the perilymph, can induce conditions that exhibit electrophysiologic similarities to those of the otoferlin-null mouse (Sultemeier \& Hoffman, 2017). Using a newlydeveloped model of spontaneous discharge we are exploring whether we can detect functional abnormalities in spontaneous discharge suggestive of synaptic modifications. These investigations are contributing to a global view of vestibular function components that are susceptible to compromise in pathogenic conditions, representing potential targets for rehabilitative strategies.

\section{The vertical computerized rotational head impulse test (crHIT-vertical)}

\section{(Abstract 24)}

Joseph Furman, MD, PhD, Jillyn Roxberg, MA, University of Pittsburgh Medical Center Ian Shirey, Alexander Kiderman, Ph.D., Neuro Kinetics, Inc.

Currently, the only means of assessing vertical semicircular canal function is the vHIT with vertical plane impulses. Head impulses are delivered manually by a clinician using one of two techniques: 1) the patient gazes right or left about $35 \mathrm{deg}$ and the head is then rotated vertically in the RALP or LARP plane, respectively; 2) the patient looks straight ahead and the head is then rotated vertically in the RALP or LARP plane. Both techniques have limitations: aligning the rotation accurately in the RALP or LARP plane is complicated and measuring vertical eye movement with video-oculography can be difficult because of goggle slippage, eyelid artifact, and non-vertical eye movement.

The crHIT-vertical mitigates the limitations of manual head impulse testing by using high speed 
video-oculography in conjunction with a computercontrolled rotation chair. crHIT-vertical accurately positions the head in the RALP or LARP plane, minimizes goggle slippage, and uses a controlled and repeatable testing environment that enables accurate calibration while considerably decreasing patient discomfort.

To determine the torque requirements of the rotation chair, a pilot study was performed to determine the minimum acceleration that could be used to reliably demonstrate vertical canals deficits. Our data suggest that a peak acceleration of $750 \mathrm{~d} / \mathrm{s}^{2}$ is required for crHIT. A multicenter trial of crHITvertical is underway to further develop this technique.

Acknowledgement: NIH grant \#2R44DC014611-02

\section{A novel protocol for tolerable human Acclimation to the cross-coupled illusion for artificial gravity}

\section{(Abstract 25)}

Kathrine N. Bretl and Torin K. Clark, Ph.D. University of Colorado Boulder - Boulder, CO

The "Coriolis" cross-coupled (CC) illusion has previously been considered the limiting factor for how fast a short-radius centrifuge could spin for artificial gravity (AG) applications. The CC illusion, caused by head tilts out of the plane of constant rotation, is highly disorienting and leads to motion sickness. Previous research has investigated methods to acclimate humans to the $\mathrm{CC}$ illusion. In our own recent efforts, we have developed a baseline personalized, incremental acclimation protocol in which subjects were exposed to the $\mathrm{CC}$ illusion with an intensity at or near their threshold (i.e., barely or not noticeable). This aimed to reduce motion sickness, improving upon previous studies' dropout rates of $\sim 30 \%$. We tested 10 subjects for 10 days, then 11 subjects for up to 50 days (always one 25-minute session per day). We found that all subjects acclimated fairly linearly (i.e., no plateau was reached), such that they did not feel any CC illusion at fairly high spin rates after just 10-15 days of acclimation training. Subjects initially felt the CC illusion at an average of 1.6 RPM (SD: $\pm 0.9, \mathrm{n}=21$ ). After 10 days, these subjects dramatically acclimated, reaching an average spin rate of 16.2 $\mathrm{RPM}( \pm 8.9)$ at which they did not feel any CC illusion. Notably, all subjects completed the protocol with minimal motion sickness, and subjects who acclimated at a slower rate than others continued to acclimate with continued exposure. In a related experiment, we tested 10 subjects in an incremental but non-personalized protocol, in which spin rate was incremented regardless of subject reporting of the presence or absence of the CC illusion. This protocol, which may be required if all astronauts are spun concurrently, was effective in acclimating humans to the CC illusion, though to a lesser extent than the personalized protocol (reaching only 11.8 \pm 3.8 vs. 16.2 RPM after 10 days). Further, motion sickness reports were higher in the non-personalized protocol with $1 / 10$ subjects unable to finish. Next, we tested whether acclimation could be retained by retesting 7 subjects following a 30 -day break. We found that these subjects were able to retain most, but not all, of the acclimation that was achieved during their initial 10-day acclimation protocol. Notably, if subjects had lost some of the acclimation during the 30-day period of no exposure, they were able to regain it at a faster rate. These findings inform the tolerability of fast spin rate, short-radius centrifugation for future implementation of $\mathrm{AG}$, a comprehensive spaceflight countermeasure.

Acknowledgements: This work was supported by a NASA Space Technology Research Fellowship. We thank J. Dixon, A. McCusker, S. Sherman, T.R. Mitchell, C. Brumley, and S. Metcalf for their assistance in data collection.

\section{The scope and scale of operational performance detriments due to motion}

\section{(Abstract 26)}

\author{
LT Adam T. Biggs Ph.D., MSC, USN \\ Naval Medical Research Unit - Dayton, $\mathrm{OH}$
}

Kyle A. Pettijohn, Ph.D.

The Henry M. Jackson Foundation for the Advancement of Military Medicine, Bethesda, MD and Naval Medical Research Unit-Dayton, $\mathrm{OH}$

LT Chad Peltier, MSC, USN, Ph.D.

Naval Submarine Research Laboratory, Groton, CT 
Jamie R. Lukos, Ph.D. and LCDR Jacob N. Norris, Ph.D.

Space and Naval Warfare Systems Center, San Diego, $C A$

Motion is one of the most pervasive performance challenges in military operations. However, while personnel are trained to handle weapon recoil and pharmacological interventions are developed to counteract motion sickness, there is no training aimed at enhancing human performance in motiondegraded environments. The assumption appears to be that motion-related detriments are unavoidable, and therefore interventions are intended to alleviate issues affecting individual readiness. These issues raise two critical questions: 1) how much does motion impair performance, and 2) why are training operations not more concerned about preparing personnel for this challenge? The present investigation utilized existing data from multiple studies with an operationally-relevant task, where a virtual reality program incorporated physical motion while participants fired a .50 caliber machine gun. Motion conditions included a no motion (baseline), relatively gentle motion (an Arleigh-Burke class destroyer), or relatively substantial motion (a rigid hull inflatable boat). Performance detriments indicated that even gentle motion reduced shot accuracy by half. The more extreme motion condition reduced accuracy to almost $10 \%$. Time to disable hostile craft tripled and nearly perfect ship disabling rates dropped to nearly $40 \%$. Moreover, these performance detriments occurred in a motion profile where the .50 caliber would be the primary weapons system, making the task highly relevant to military operations. These impairments demonstrate how substantial the problem is and how imperative it is that new training methods be developed to help military personnel address the challenges of performing in a moving environment.

\section{Reference}

Author, (Year). Journal \#(\#).

Acknowledgement: Funded by the Office of Naval Research under work unit numbers H1515 and H1602.

\section{Vestibular modulation of hippocampal and striatal function}

\section{(Abstract 27)}

Paul F. Smith ${ }^{1}$, Martin Hitier ${ }^{1,2}$, Yanfeng Zhang ${ }^{1}$, Go Sato $^{1}$, Lucy Stiles ${ }^{1}$, Yiwen Zheng ${ }^{1}$, John Reynolds ${ }^{1}$, Ruth Napper ${ }^{1}$, Stephane Besnard ${ }^{2}$, Alice Benoit ${ }^{2}$, Bruno Philoxene ${ }^{2}$, Bruno Truchet $^{3}$ and Bruno Poucet $^{3}$

${ }^{1}$ Dept. Pharmacology and Toxicology, Dept. Anatomy, School of Biomedical Sciences, the Brain Health Research Centre, and Brain Research New Zealand, University of Otago, Dunedin, New Zealand

${ }^{2}$ Université de Normandie, INSERM U 1075 COMETE, Caen France

${ }^{3}$ Aix Marseille Université, CNRS, Marseille, France.

Numerous studies have now demonstrated that lesions to, or stimulation of, the vestibular system, can alter neuronal activity in the hippocampus (HPC) and striatum (STR). Vestibular modulation of these brain regions, is known to be involved in the development of both spatial memory and motor plans for action. Nonetheless, we still have a very limited understanding of how vestibular input modulates HPC and STR activity. We have selectively electrically stimulated different vestibular sensors and recorded electrophysiological activity in the HPC, and investigated the effects of bilateral vestibular loss (BVL) on HPC long-term potentiation (LTP), and N-methyl-D-aspartate (NMDA) receptors involved in mediating LTP. Responses could be evoked by stimulation of all semi-circular canals as well as the utricle and saccule; however, the patterns of activation varied by sensor, with larger responses evoked from the otoliths. The responses were bilateral, greater than $20 \mathrm{~ms}$ in latency, with some lateralization. Compared to controls, BVL, at 30 days post-op. caused increased potentiation of the HPC population spike without any change in the field excitatory post-synaptic potential (fEPSP), ('ES-potentiation'), which indicates a lack of inputspecificity and is associated with poorer spatial memory. This correlated with a significant increase in the density of NMDA receptors, and in the number of neurons expressing NMDA receptors, at 7 and 30 days post-BVL. In other studies we have explored the effects of electrical stimulation of the rat vestibular labyrinth on single neuron activity, c-Fos 
expression and neurotransmitter release in the STR. We have found a small, circumscribed number of STR neurons that responded with increases in firing; however, c-Fos expression decreased in a currentdependent manner, across the entire STR. The number of STR neurons expressing NMDA receptors decreased at 7 but not 30 days post-BVL. Using in vivo microdialysis, we found that vestibular stimulation decreased the release of serine, threonine and taurine in the STR; although dopamine (DA) itself was not significantly affected, DA metabolism was decreased. These studies suggest that vestibular information is transmitted to both the HPC and STR, and that both brain regions are likely to be affected following vestibular dysfunction, separately and in their interactions.

\section{The activity of the Purkinje cells in the vestibular cerebellum during active and passive self-motion}

\section{(Abstract 28, Poster Location T)}

\section{Omid Zobeiri, Kathleen Cullen}

The ability to distinguish between self-generated (reafference) vs. externally-applied (exafference) sensory signals is fundamental for ensuring accurate motor control as well as perceptual stability. This is particularly evident in the context of the vestibular system, in which the same central vestibular neurons that receive direct afferent input also directly project to motor centers to control vestibulo-spinal reflexes. Notably, while vestibulo-spinal reflexes are essential for providing a robust postural response to unexpected vestibular stimuli, they are counterproductive when the goal is to make active head movements. Previous studies by our group have shown that vestibular-only (VO) neurons in the vestibular nuclei at the first central stage of processing preferentially code vestibular exafference in both monkeys and mice. However, the neural mechanism underlying the suppression of vestibular reafference is unknown. Accordingly, here, to investigate the neuronal basis of vestibular reafferent suppression, we recorded from Purkinje cells in the vestibular cerebellum (anterior vermis, lobules IVV). Single-unit extracellular recordings were made in rhesus monkeys during comparable active and passive head rotational movements, and Purkinje cell spikes were detected via a semi-automated clustering algorithm. Our analysis of responses during passive motion first revealed robust simple spike responses to head motion with some neurons showing bidirectionality, others showing directional rectification, and others showing unidirectionality. Next, the comparison of neuronal responses during passive and active head rotations demonstrated that simple spike responses were markedly attenuated $(\sim 80 \%)$ across our population of Purkinje cells during active versus passive rotations. We hypothesized that sensory-motor convergence of the neck motor and proprioceptive inputs in the vestibular cerebellum plays a critical role in the computations required to generate a reafferent vestibular suppression signal. Our results show that indeed neurons in the anterior vermis can respond to passive proprioceptive stimulation. We also tested whether Purkinje cells responded to neck motor signals (i.e., efference copy) by recording responses as the monkey attempted to move its head, while we simultaneously applied a break that prevented head motion relative to space and found that most neurons were also modulated by neck efference copy. However, the proprioceptive and efference copy related responses varied considerably across neurons, and the summation of these inputs could not explain a given Purkinje cell's attenuated response to active head motion. Taken together, these results provide new insights into the computations performed by Purkinje cells in anterior vermis that underlie the cancellation of vestibular reafference.

\section{Space and the Vestibular System: What Have We Learned?}

\section{(Abstract 29)}

Laurence R. Young, Sc.D.

Massachusetts Ins

Cambridge, Massachusetts Institute of Technology

The very absence of sustained gravito-inertial force during orbital space flight naturally produces profound effects on the graviceptors in the body including the vestibular system. At the outset of the space age doubts were present concerning the viability of humans in space, including their ability to sleep, exercise fine motor control, and resist 
disabling motion sickness. From the earliest reports of disorientation and symptoms of motion sickness - first with cosmonauts and then with astronauts the focus of research shifted to the underlying responses of the vestibular systems and its interaction with visual and proprioceptive signals. Space sickness was recognized as a common malady and means to predict it, limit it, and treat it were investigated. Artificial Gravity, with its inevitable Coriolis forces, produced novel and disturbing vestibular stimuli as well as the desired gravitoinertial forces. This presentation provides an overview of sixty years of vestibular research in space and examines the key questions as well as the current understanding and theories of space motion sickness and vestibular adaptation. Emphasis is made on the responses of the semicircular canals and otoliths to rotatory and linear acceleration, and to the influence of non-vestibular signals in human spatial orientations and eye movements.

\section{The role of vestibular noise in velocity storage dynamics}

\section{(Abstract 30, Poster Location E)}

Benjamin Benac ${ }^{\mathrm{a}, \mathrm{b}}$, Sirine Nouri ${ }^{\mathrm{a}, \mathrm{b}}$, Richard F. Lewis, M.D. ${ }^{a}$, Daniel M. Merfeld, Ph.D. ${ }^{\mathrm{c}}$, Faisal Karmali, Ph.D. ${ }^{\mathrm{a}}$

a Jenks Vestibular Physiology Lab, MEEI; Harvard Medical School, Boston MA

${ }^{\mathrm{b}}$ Ecole Centrale Lyon, Lyon, France

'The Ohio State University, Columbus OH and Naval Medical Research Unit-Dayton $\mathrm{OH}$

The velocity storage time constant is an important clinical parameter which varies with age, pathology and stimulus amplitude. It has been hypothesized that Bayesian optimal processing determines velocity storage dynamics based on the statistics of vestibular noise and experienced motion. Specifically, while a longer time constant would be advantageous because this would make the VOR accurate over a longer period of time, it has been argued that this would result in the integration of noise by the velocity storage mechanism, which would result in drift and make the VOR less precise (1). Through experiments and modeling, we have recently found evidence to support the hypothesis that the brain determines the time constant based on vestibular noise to determine the optimal tradeoff between being accurate and being precise. First, we found that age-dependent changes in the VOR are explained by a Bayesian optimal velocity storage model responding to death of motion-sensing hair cells (2). Specifically, we found that the temporal dynamics of the reflex as a function of age are predicted $(r=0.93, p 0.001)$ by a Kalman filter model which determines the optimal behavioral output when the sensory signal-to-noise characteristics are degraded by death of the transducers. Second, we have found that these models predict reduced velocity storage time constants for unilateral lesions. Third, we found that Bayesian processing explains the decrease in velocity storage time constant as stimulus velocity increases; these predictions, which were experimentally verified, were made using recent measurements that showed increasing VOR and perceptual variability with angular velocity (3). Together, these results complement existing work showing optimal, static changes in vestibular responses due to aging and lesions. These results also provide the groundwork for investigation of Bayesian, dynamic control of the VOR due to variations in vestibular function.

\section{Reference}

1. Karmali, F. \& Merfeld, D. M. A distributed, dynamic, parallel computational model: the role of noise in velocity storage. J Neurophysiol 108, 390405 (2012).

2. Karmali, F., Whitman, G. T. \& Lewis, R. F. Bayesian optimal adaptation explains age-related human sensorimotor changes. J Neurophysiol 119, 509-520 (2018).

3. Nouri, S. \& Karmali, F. Variability in the Vestibulo-Ocular Reflex and Vestibular Perception. Neuroscience 393, 350-365 (2018).

Acknowledgements: Funded by NIH/NIDCD R03DC013635 and R01 DC-013069. 


\section{Neural Representations of Natural Self-Motion: Implications for Perception \& Action}

\section{(Abstract 31)}

Dr. Kathleen E. Cullen, Professor Department of Biomedical Engineering

The Johns Hopkins University, Baltimore, MD 21205, U.S.A.

A fundamental question in neuroscience is how does the brain compute accurate estimates of our selfmotion relative to the world and orientation relative to gravity in everyday life. In this talk, I will describe recent findings from my laboratory's research that have addressed this question and provided new insight into how vestibular pathways encode selfmotion information to ensure accurate perception and motor control. First, we have recently examined the statistics of natural self-motion signals experienced by mice, monkeys, and humans, and then explored the neural coding strategies used by early vestibular pathways. Focusing on the relationships between neural variability, detection thresholds, and information transmission, our findings have revealed that two distinct sensory channels represent vestibular information at the level of the vestibular periphery. Notably, more regularly discharging afferents have better detection thresholds and use rate coding, while more irregular afferents take advantage of precise spike timing (i.e, temporal coding) and are better optimized for processing natural vestibular stimuli. Our research has also established that the neurons at the first central stage of vestibular processing are substantially less sensitive to active motion. Notably, this ability to distinguish between active and passive motion is not a general feature of early vestibular processing, but is instead a characteristic of a distinct group of neurons known to contribute to postural control and spatial orientation. Our most recent studies further indicate that multimodal integration within the vestibular cerebellum is required for this cancellation of self-generated vestibular information from the subsequent computation of orientation and posture control. Moreover, when unexpected vestibular inputs become persistent during active motion, this mechanism is rapidly updated to reenable the vital distinction between active and passive motion to ensure the maintenance of posture and stable perception.

Acknowledgements: Funded by NIH/NIDCD R01DC2390, the Canadian Institutes of Health Research (CIHR), Natural Sciences and Engineering Research Council of Canada (NSERC), and Canada Foundation for Innovation (CFI).

\section{Perceived timing of multisensory events during self-motion}

\section{$\underline{\text { (Abstract 32) }}$}

\author{
Michael Barnett-Cowan \\ Department of Kinesiology, University of Waterloo, \\ Waterloo, Canada
}

Multisensory stimuli originating from the same event can be perceived asynchronously due to differential physical and neural delays. The transduction of and physiological responses to vestibular stimulation are extremely fast, suggesting that other stimuli need to be presented prior to vestibular stimulation in order to be perceived as simultaneous. There is, however, a recent and growing body of evidence from our lab and others (see Barnett-Cowan 2013 for review), which indicates that the perceived onset of vestibular stimulation is slow compared to the other senses, such that vestibular stimuli need to be presented prior to other sensory stimuli in order to be perceived synchronously. In this talk I will review this literature and explore possible explanations that can account for vestibular perception being slow, while acknowledging that it is really the onset of selfmotion estimated from multiple senses that is perceived as being relatively slow. I will also present our most recent studies focused on the perceiving timing of multisensory events during a fall (Lupo and Barnett-Cowan, 2017; 2018) which reveal 1) perceptual delays of fall onset relative to other external events, 2) distorted representations for the duration of external events that are coincident with a fall, and 3) increased fall perception delays and distortions of duration estimation in the elderly population.

\section{References}

1. Barnett-Cowan, M. (2013). Vestibular perception is slow: a review. Multisensory research, 26(4), 387-403. 
2. Lupo, J., \& Barnett-Cowan, M. (2017). Perceived timing of a postural perturbation. Neuroscience letters, 639, 167-172.

3. Lupo, J., \& Barnett-Cowan, M. (2018). Impaired perceived timing of falls in the elderly. Gait \& posture, 59, 40-45.

Acknowledgements: Funded by NSERC RGPIN-05435-2014

\section{Comparison of vestibular perceptual thresholds in pitch versus roll tilt}

\section{(Abstract 33)}

Kadambari Suri and Torin K. Clark, Ph.D.

University of Colorado - Boulder, Boulder, CO

Vestibular perceptual thresholds quantify human sensitivity to perception of small motions in the dark. Thresholds relate to postural stability, manual control nulling performance, falls, and tend to increase with age (1). Further, they appear to be altered in patient populations (e.g., vestibular migraine patients, bilateral defectives (2), or elderly individuals with an increased risk of falling) thus serving as a potential diagnostic assessment. Thresholds have been well quantified in healthy humans across a range of axes and have been shown to vary as a function of stimulus frequency (2). For example, Z-axis (inferior-superior earth-vertical) translation thresholds are higher compared to Y-axis (inter-aural earth-horizontal) thresholds $(1,2)$. Similarly yaw rotation thresholds have been observed to differ from those in pitch and roll (3). Less attention has been paid to threshold differences based upon the axis of tilt. Roll tilt thresholds have been well quantified $(1,2)$, but pitch tilt much less so. Here we quantify pitch tilt thresholds and compare to those in roll tilt in the same group of subjects, across a range of stimulus frequencies, using standard, modern threshold approaches. Specifically, whole-body tilt motions were produced using our Tilt Translation Sled without the translation axis activated. The axis of tilt was located at the subject's head, which was fixed in a custom head restraint. After each unidirectional tilt motion in the dark, subjects verbally reported the perceived direction (e.g., forward or backward for pitch tilt) in a forced-choice, direction-recognition task.
Responses were fit using a standard cumulative Gaussian psychometric curve to estimate the 1 sigma threshold. Ten subjects performed blocks of 200 trials for each tilt axis (roll or pitch) and stimulus frequency $(0.15,0.2,0.5$ or $1 \mathrm{~Hz})$ presented in a counterbalanced order. As previously observed for roll, tilt angle thresholds increased at lower frequencies, but stabilized around 0.15-0.2 Hz. Pitch tilt thresholds, across each of the frequencies we tested, were observed to be similar to, but slightly higher than, roll tilt thresholds. Specifically the geometric mean threshold for pitch tilt (versus roll tilt) was $1.66^{\circ}\left(1.47^{\circ}\right)$ for $0.15 \mathrm{~Hz}, 1.61^{\circ}\left(1.46^{\circ}\right)$ for $0.2 \mathrm{~Hz}, 0.99^{\circ}\left(0.96^{\circ}\right)$ for $0.5 \mathrm{~Hz}$, and $0.51^{\circ}\left(0.47^{\circ}\right)$ for $1 \mathrm{~Hz}$. To our knowledge this is the first time pitch tilt thresholds have been quantified across a range of frequencies, providing a comparative baseline of healthy subjects.

\section{References}

1. Bermudez Rey, et al. (2016) Frontiers or Neurophysiology 7.

2. Valko, et al. (2012) Journal of Neuroscience 32.

3. Benson, et al. (1989) Aviation Space and Environmental Medicine 60.

\section{Influence of heading separation on visual and vestibular direction perception and common causation.}

\section{(Abstract 34)}

\section{Authors: Benjamin T. Crane and Raul Rodriguez}

Visual and vestibular or inertial cues are used to determine heading direction. Visual cues can represent either self or environmental motion and when environment motion is present, such being in a crowd, may be offset from vestibular cues. In a fixed in environment visual and inertial cues have a common causation and should be integrated. In a moving environment, visual and inertial cues no longer have common causation and should be perceived independently. Effects of directionally offset visual and inertial cues on heading perception and common causation were examined in four normal subjects. Both stimuli consisted of $2 \mathrm{~s}$ of motion with a similar motion profile. The visual stimulus consisted of a star field with coherence reduced to $70 \%$ to make reliability similar to inertial 
motion. Trial blocks included 12 possible visual and inertial headings which covered the full $360^{\circ}$ range in the horizontal plane in $30^{\circ}$ increments. All possible heading combinations were presented in random order with 144 stimuli in each trial block. After each stimulus, subjects used a mechanical dial to report the perceived direction of the visual or inertial heading, and buttons to report if the headings were the same or different. Six trial blocks were performed in each subject, in 3 blocks inertial heading was reported and in the other 3 visual heading was reported. In all 6 blocks subjects reported if headings were the same or different. With a $0^{\circ}$ offset subjects reported they were the same in $68 \%$ of trials. However, when aligned in a cardinal direction the rate was $95 \%$, while only $55 \%$ for alignment in other directions. With a $30^{\circ}$ offset $47 \%$ were similar, and $60^{\circ}$ offset $14 \%$, and less than $2 \%$ for larger offsets. Visual heading perception was not influenced by the inertial heading. Inertial headings were biased towards the visual heading with offsets of $30-120^{\circ}$ (T-test p 0.01). With offsets of 30, 90, and 120 the inertial heading was biased 8.1 to $8.6^{\circ}$, with a $60^{\circ}$ offset the bias was largest at $11.5^{\circ}$. Biases were larger indicating better multi-sensory integration when common causation was reported, but still occurred in trials where there was no common causation. Thus, visual motion influences inertial heading perception even when headings are displaced beyond the range in which they are perceived to have common causation. These experiments demonstrate visual and inertial headings are most likely to be perceived as the same when they are aligned with a cardinal direction and that integration of visual and inertial cues is independent of common causation perception. This makes visualvestibular integration unique compared with other types of multisensory integration.

Support: R01 DC013580

\section{Modeling neural adaptation of spatial orientation perception in humans to altered gravity}

\section{(Abstract 35)}

Jordan B. Dixon, MS, Nisar Ahmed, Ph.D., Torin

K. Clark, Ph.D.

University of Colorado - Boulder, Boulder, CO
The immense challenges associated with space exploration are amplified by the exposure of human physiology to novel environments. Particularly, when transitioning to altered gravity environments, the brain must reinterpret sensory cues in order to properly estimate spatial orientation, for appropriate motor control. The effects of gravity transitions include postural and locomotion impairments, manual control decrements, motion sickness, and errors in perception of spatial orientation (1). To better understand the potential neural mechanisms involved, we have developed the first quantitative, computational model for adaptation of human orientation perception to altered gravity environments.

We extended the well-validated "observer" model for three-dimensional, passive, vestibular spatial orientation perception (2), by allowing parameters within the central nervous system's (CNS) "internal models" to vary over time. Specifically, we implemented the hypothesis that the sensory conflict signals within the model are used to update internal parameters. In order to best update these, we implemented an approach in which the CNS assesses "multiple hypotheses" for internal parameters, computing a sensory conflict for each. The combined and normalized conflict signals from each hypothesis are compared by an online supervisor that then adjusts internal parameters in an effort to best interpret the incoming stimuli. For preliminary development of the model, the supervisor was constrained to adjust only the internal estimate of the magnitude of gravity, suitable for the altered gravity scenarios considered. We explore the potential of Bayesian approaches to "optimally" update internal parameters, given noisy sensory measurements. The extended model is simulated to predict orientation perception responses from a preliminary experiment (3), in which humans were exposed to a sustained altered gravity environment via centrifugation. With no knowledge of the actual gravity environment, the computational model properly adjusts its internal estimate of the magnitude of gravity, reduces sensory conflict, and improves tilt perception, similar to that observed experimentally. In conclusion, we explored the potential neural mechanisms of sensorimotor adaptation to altered gravity through implementing and simulating a computational model. This approach may be used to model other scenarios requiring sensorimotor adaptation (e.g., vestibular dysfunction). 


\section{References}

1. Reschke and Clément (2018) Current Pathobiology Reports 6.

2. Merfeld et al. (1993) Journal of Vestibular Research 3.

3. Galvan-Garza, et al. (2018) Journal of Neurophysiology 120.

\section{Roll tilt self-motion direction discrimination: First evidence for perceptual learning}

\section{(Abstract 36, Poster Location L)}

Klaus M.P. ${ }^{1}$, Schöne C.G. ${ }^{1,2,3}$, Hartmann M. ${ }^{1,3}$, Merfeld D.M. ${ }^{4}$, Schubert M.C. ${ }^{5,6}$, \& Mast F.W. ${ }^{1}$ ${ }^{1}$ Department of Psychology, University of Bern, Bern, Switzerland,

${ }^{2}$ Department of Otorhinolaryngology, Head and Neck Surgery, University Hospital Bern, University of Bern, Bern, Switzerland,

${ }^{3}$ Faculty of Psychology, Swiss Distance University, Brig, Switzerland,

${ }^{4}$ The Ohio State University, Columbus, Ohio, USA, ${ }^{5}$ Laboratory of Vestibular Neuro Adaptation, Department of Otolaryngology - Head and Neck Surgery, Johns Hopkins University, Baltimore, USA, ${ }^{6}$ Department of Physical Medicine and Rehabilitation, Johns Hopkins University, Baltimore, USA

Perceptual learning, the ability to improve the sensitivity of sensory perception through training, has been shown to exist in all sensory modalities but the vestibular system. Previous studies have failed to demonstrate an improvement of passive self-motion thresholds in the dark for yaw rotation and y-translation, but subjects improved for the same motions in the light (Hartmann et al., 2013). The goal of the present study was to investigate whether vestibular perceptual learning in the dark would occur when there is a simultaneous otolith and semicircular canal input, as is the case with roll tilt motion stimuli. Blindfolded subjects $(\mathrm{N}=10)$ trained a direction discrimination task with $0.2 \mathrm{~Hz}$ roll tilt motion stimuli for 6 days, for a total of 1800 trials. Before and after training, motion thresholds were measured for the trained motion $(0.2 \mathrm{~Hz}$ roll tilt) and for three transfer conditions ( $1 \mathrm{~Hz}$ roll tilt, $0.2 \mathrm{~Hz}$ pitch, $0.2 \mathrm{~Hz}$ y-translation). Performance before and after training was analyzed using a
Bayesian hierarchical logistic regression. We found that sensitivity was increased after training in the 0.2 $\mathrm{Hz}$ roll tilt condition (trained motion) and in the $1 \mathrm{~Hz}$ roll tilt condition (Transfer condition 1), but not in the other motion conditions. This is the first demonstration of perceptual learning of passive selfmotion direction discrimination in the dark using low frequency roll tilt motion stimuli. Surprisingly, we found that a control group with no training within this 6 day period also improved in the $1 \mathrm{~Hz}$ roll tilt condition but not in any other motion condition. It is possible that high frequency roll tilt motion $(1 \mathrm{~Hz})$ leads to faster learning and does not require extensive training, which requires further study to evaluate. The results have potential therapeutic consequence as higher $0.2 \mathrm{~Hz}$ roll tilt thresholds have been associated with worse performance in balance tests that may increase morbidity (Karmali et al., 2017, Bermúdez Rey et al., 2016).

\section{References}

1. Bermúdez Rey et al. (2016). Frontiers in Neurology 7(162).

2. Hartmann et al (2013). Experimental Brain Research 226(231-240).

3. Karmali et al (2017). Frontiers in Neurology $8(578)$.

\section{Gulf war illness in US veterans is associated with otolith hypofunction and decreased brain blood flow}

\section{(Abstract 37)}

\author{
J. M. Serrador ${ }^{1,2}$, S.J. Wood ${ }^{3,4}$ \\ ${ }^{1}$ War Related Illness \& Injury Study Center, NJ VA \\ Health Care System, East Orange, NJ \\ ${ }^{2}$ Rutgers Biomedical Health Sciences, Newark, NJ \& \\ National University of Ireland Galway, Ireland \\ ${ }^{3}$ Azusa Pacific University, Azusa, CA \\ ${ }^{4}$ NASA Johnson Space Center, Houston, TX.
}

Gulf War Illness (GWI) is a chronic fatigue like syndrome that plagues almost $25 \%$ of the 700,000 veterans that returned form operation Desert Storm/ Desert Shield in 1990-91 and presents with cognitive problems and brain fog. To examine a possible role for vestibular inputs in this syndrome, we recruited a group of 9 veterans with GWI who were participating in an autonomic study. We measured ocular torsion 
in these individuals during a $+/-20 \mathrm{O}$ sinusoidal roll tilt at $0.05 \mathrm{~Hz}$. We also measured beat-by-beat blood pressure using a finger cuff and cerebral blood flow velocity using transcranial Doppler during a sit to stand test. We found that ocular torsion was significantly correlated to the decrease in cerebral blood flow when standing ( $\mathrm{R}=0.47, \mathrm{P} 0.05)$ with lower torsion being associated with greater decreases in cerebral flow. In contrast, there was no relationship between torsion and blood pressure or heart rate while standing. These data suggest that veterans with GWI and poor otolith function (indicated by low ocular torsion) demonstrate greater drops in brain blood flow when standing. Since maintenance of brain blood flow is essential to cognitive function, reduced brain blood flow upright might contribute to cognitive impairment. These data are consistent with the theory that otolith mediated inputs of gravity, help to dilate your cerebral vessels when upright and maintain cerebral blood flow when your head is above your heart. Without intact otolith function, this dilation may be impaired and these individuals may be suffering from cerebral hypoperfusion. Supported by CDMRP grant W81XWH-14-1-0598 (Serrador).

\section{Modification of vestibular calyx membrane properties by different groups of efferents: a requirement for normal afferent function}

\section{(Abstract 38)}

Soroush G. Sadeghi

Center for Hearing and Deafness and Neuroscience Program, State University of New York at Buffalo, Buffalo, NY

Stimulation of vestibular efferents increases the resting discharge and decreases the sensitivity of afferent fibers. The amplitude of efferent-mediated responses increases with the increase in the irregularity of the afferent resting discharge, a property attributed to the calyx afferent terminal. Such calyx afferent terminals contain voltage sensitive potassium channels that if left open at resting membrane potentials, result in a strong spike frequency adaptation that precludes spontaneous firing. In fact, under in vitro conditions where efferents are damaged, most afferents do not have a resting discharge and step depolarization of calyces results in firing of a single action potential. Recent studies in our lab has shown that efferent inputs could modulate the activity of these channels in order to modify membrane properties and adjust the firing rate of afferents. Patch clamp in vitro recordings from calyces in the cristae provide evidence that activation of metabotropic receptors increases the speed and sensitivity of responses of calyx terminals through changes in the activity of different potassium channels. While previous studies have focused on the function of cholinergic efferents, we provide evidence of existence of GABA-B receptors on calyx terminals that function in concert with muscarinic acetylcholine receptors (mAChR) to decrease the activity of KCNQ channels and calcium-activated potassium channels, respectively. Consistent with these findings, we found that inhibition of efferents during in vivo extracellular single unit recordings from afferent fibers inhibits the resting discharge of irregular afferent fibers. Finally, we found that the synchronized activity of irregular fibers by vestibular sensory evoked potentials (VsEP) could be modulated by intracochlear injection of agonists and antagonists of efferent receptors. Together, these findings provide strong evidence that cholinergic and GABAergic efferents are required for normal activity of irregular afferents and could modulate the sensitivity and latency of responses of irregular afferents.

These studies were funded by NIH-NIDCD grant 5R03DC015091 and a research grant from the American Otological Society (AOS).

\section{Nav1.6 channels mediate persistent and resurgent sodium currents in vestibular calyx afferent terminals}

\section{(Abstract 39)}

Frances L. Meredith Ph.D., Karen Dockstader, Alexander Arne, Katherine J. Rennie Ph.D. University of Colorado School of Medicine, Aurora CO 80045

Primary vestibular afferent neurons can fire action potentials at high rates and modulation of firing patterns is key for coding of vestibular signals. Afferent firing patterns vary with the position of 
terminal endings in vestibular neuroepithelia, but the significance of these differences is unclear (Eatock and Songer 2011). We recently characterized transient $\mathrm{Na}+$ currents in zonally-identified vestibular afferent subtypes (Meredith and Rennie 2018). Transient Na+ currents in mature calyces of crista peripheral zones weresubstantiallyreducedby 4,9 -anhydrotetrodotoxin (4,9-ah-TTX), an analog of tetrodotoxin (TTX) that selectively blocks Nav1.6 channels. Immature calyces and mature calyx-only terminals located in central regions of the crista were less sensitive to 4,9-ah-TTX, suggesting that Nav1.6 channels contribute minimally to $\mathrm{Na}+$ currents in these afferents (Meredith and Rennie 2018). In addition to transient currents, Nav1.6 channels can mediate persistent and resurgent $\mathrm{Na}+$ currents in a variety of cell types. These atypical currents are much smaller than the transient current that flows through $\mathrm{Na}+$ channels and are associated with neurons that have the capacity for rapid spontaneous firing. In this study we investigated the contribution of persistent and resurgent components to $\mathrm{Na}+$ currents in afferent terminals of developing and mature cristae.

Whole cell patch clamp recordings were obtained from calyces in transverse slices of gerbil cristae in two age groups: immature (postnatal day $(\mathrm{P}) 5$ to P14) and mature (P24 to P44). Specific voltage protocols were designed to evoke transient, resurgent and persistent $\mathrm{Na}+$ currents in voltage clamp. Action potentials were recorded in current clamp. Fluorescent dye fills were used in some cells to identify calyx-only and dimorphic afferents. Immunohistochemistry was performed using an antibody to target Nav1.6 channels and revealed nodal and terminal staining for Nav1.6 in mature cristae. A small TTX-sensitive persistent current was often observed in calyx recordings following the transient $\mathrm{Na}+$ current. ATX-II, a sea-anemone toxin that decreases the inactivation of $\mathrm{Na}+$ channels, slowed $\mathrm{Na}+$ current inactivation and boosted persistent currents in all immature and mature calyces tested. However, 4,9-ah-TTX reduced the enhanced $\mathrm{Na}+$ current in mature calyces only. In peripheral zone calyces, 4,9-ah-TTX

Significantly reduced the height and increased the width of action potentials. A TTX-sensitive resurgent current was evoked in voltage clamp at membrane potentials between -50 and $-10 \mathrm{mV}$ following brief $(10 \mathrm{~ms})$ depolarizations of the calyx membrane from -90 to $+20 \mathrm{mV}$. In summary we have identified persistent and resurgent $\mathrm{Na}+$ currents in calyx- bearing primary vestibular afferents and shown that Nav1.6 channels contribute to action potential firing in mature calyces of crista peripheral zones.

\section{References}

1 Eatock RA, Songer JE. Vestibular hair cells and afferents: two channels for head motion signals. Annu Rev Neurosci 34: 501-534, 2011. 2 Meredith FL, Rennie KJ. Regional and developmental differences in $\mathrm{Na}+$ currents in vestibular primary afferent neurons. Front Cell. Neurosci. 12: 423, 2018.

\section{Infrared Photo-sensitivity in the vestibular neuroepithelium is modulated by TRPV4}

\section{(Abstract 40)}

Federica M. Raciti

Dept. of Cellular Physiology and Molecular Biophysics, University of Miami, Miami, FL

Weitao Jiang, $\mathrm{PhD}$.

Dept. of Biomedical Engineering, University of Miami, Miami, FL

Suhrud M. Rajguru, PhD.

Dept. of Otolaryngology, University of Miami, Miami, FL

Dept. of Biomedical Engineering, University of Miami, Miami, FL

Dept. of Cellular Physiology and Molecular Biophysics, University of Miami, Miami, FL

Introduction: Pulsed infrared radiation (IR) is being investigated as a non-invasive technique for altering activity of excitable cells such as nerve and muscle. However, the mechanisms of action of IR are poorly understood. Previous studies suggest that IR induced intracellular $[\mathrm{Ca} 2+]$ changes as a result of activation of temperature-dependent Transient Receptor Potential (TRP) channels. In the present work, we investigated the mechanisms underlying IR responses in the vestibular neuroepithelium focusing on the role of the thermosensitive TRP channels. We hypothesized that IR-activation of TRPV4 channels modulates $[\mathrm{Ca} 2+] \mathrm{i}$ leading to glutamate release from vestibular hair cells and the observed excitatory and inhibitory post-synaptic responses. 
Materials and Methods: The University of Miami Institutional Animal Care and Use Committee approved all procedures. Bilateral eye movements were recorded and characterized during pulsed IR stimulation of vertical semicircular canals in vivo in a rat model to assess the activity of the vestibular system (ISCAN Inc, Woburn, MA). Results were analyzed using custom MATLAB program. IR at $1860 \mathrm{~nm}(200 \mu \mathrm{s}, 200 \mathrm{~Hz}$, various radiant exposures) was directed at the vestibular neuroepithelium via a $200 \mu \mathrm{m}$ dia. optical fiber. IR evoked eye movements were measured before and after the neurotransmission was impaired upstream by an acute treatment with Neomycin $(100 \mathrm{mM})$, causing hair cells loss, or downstream by perfusion of CNQX $(100 \mu \mathrm{M})$, a competitive AMPA/kainate receptor antagonist. Furthermore, the IR response was also recorded prior to and after reducing the temperature below the activation threshold of the TRPV4 $\left(<26^{\circ} \mathrm{C}\right)$ with perfusion of temperature-controlled artificial perilymph. TRPV4 channels were targeted pharmacologically with specific blockers (GSK2193874 and HC067047) perfused at different concentrations.

Results and Discussion: The IR evoked amplitude of eye movement reduced significantly following treatment with both CNQX and Neomycin. The amplitude of IR-evoked eye movement, also, reduced significantly with temperatures lower than TRPV4 activation threshold as well as after the perfusion of TRPV4 channel blockers. The eye movement recovered at the physiological temperatures and after washout of the compounds suggesting that TRPV4 channels play an important role in IR activation of the vestibuloocular motor pathway.

Conclusion: These results suggest that IR stimulation primarily affects vestibular hair cells and that TRPV4 channels within the vestibular neuroepithelium drive the photothermal responses.

Funding: NIH NIDCD 1R01DC008846 and 1R01DC013798

\section{Vestibular sensory cell development and regeneration: the potential for recovery from hair cell and afferent loss}

\section{(Abstract 41)}

\author{
Jennifer Stone, Brandon Cox, Rémy Pujol, James \\ Phillips, Ruth Anne Eatock
}

The discovery that non-mammalian vertebrates regenerate vestibular hair cells and recover vestibular reflexes after damage raised enthusiasm that similar innate abilities could exist in mammals. Two decades of research showed that mature rodents and perhaps humans replace $\sim 20 \%$ of vestibular hair cells after damage and exhibit a slow rate of cellular turnover during normal conditions. This renewal is limited to type II hair cells; there is no evidence that type I hair cells are replaced in either situation. Our lab, in collaboration with other investigators, studies the cellular and molecular mechanisms underlying hair cell regeneration and turnover in mice, using engineered mouse lines to fate-map cells and to delete genes that potentially control these processes. Cell-tracking demonstrated that supporting cells phenotypically convert into type II hair cells after destruction of both type I and II hair cells. Deletion of the transcription factor Atoh1 in supporting cells prior to damage abolished this process and prevented any regeneration. We examined new type II hair cells and their associated afferent nerves following hair cell destruction in utricles and horizontal ampullae. Regenerated hair cells acquired several features characteristic of mature type II hair cells, including basolateral processes and short, thin stereocilia with proper bundle orientation. New hair cells also acquired voltage-gated $\mathrm{K}$ conductances typical of type II hair cells, and they exhibited receptor potentials upon bundle displacement, although potentials were smaller than those previously recorded in type II hair cells. Oddly, regenerated hair cells retained voltage-gated $\mathrm{Na}$ currents typical of immature hair cells, even after long recovery periods. Afferent fibers were largely lost from the macula within days of damage, with calyceal terminals becoming swollen and filled with mitochondria prior to their disappearance. However, ganglion neurons survived, and by 4 weeks postdamage, afferent neurites had re-entered the epithelium and ribbon synapses had formed between 
regenerated hair cells and afferent nerves. By 6 weeks, synapses had reached mature density and distribution. Despite signs of maturation and reconnection with vestibular nerve afferents, our initial investigations indicate that vestibular function was not restored, as reflected by gross motor behaviors, horizontal VOR, and rotation-evoked cFos labeling in the brainstem. Future studies will address why only a subpopulation of vestibular hair cells is regenerated. Further, we will determine if the natural capacity for hair cell and neural regeneration in adult mice can be enhanced by genetic manipulations and if vestibular function is restored.

\section{Using vestibular efferent synaptic mechanisms to probe efferent function}

\section{(Abstract 42)}

J. Chris Holt, Ph.D.

University of Rochester, Rochester, NY

The mammalian efferent vestibular system (EVS) begins as a modest number of bilateral, multipolar neurons in the dorsal brainstem. The axons of contralateral vestibular efferent neurons (VENs) cross the midline where they converge with ipsilateral VENs to innervate the peripheral vestibular end organs by way of cranial nerve VIII. Within each end organ, VENs undergo extensive branching and give rise to thousands of prominent, vesiculated varicosities abutting afferent terminals and hair cells. VENs, by virtue of this very anatomy, offer the CNS with a direct line to both sides of the first synapse in vestibular transduction. Activation of the mammalian EVS, at least under experimental conditions, profoundly modulates the resting discharge and sensitivity of vestibular afferents along multiple time scales. Despite these well characterized anatomical and neurophysiological observations, the functional underpinnings for how and when the CNS utilizes the EVS in mammals has remained rather elusive. We have long submitted that pharmacological characterization of mammalian EVS actions is one of the first steps for identifying how VENs modify afferent response metrics. These pharmacological tools, once identified, should also be of great utility in evaluating EVS function in behaving animal models. While immunohistochemical data in mammalian VENs have implicated the neurotransmitters acetylcholine (ACh) and calcitonin gene-related peptide (CGRP), direct pharmacological evidence for either's role in the response of mammalian vestibular afferents to efferent stimulation had been lacking. This talk will summarize our recent work describing the pharmacological characterization of synaptic mechanisms governing mammalian EVS actions in a novel, in vivo mouse preparation. We will present evidence that the mammalian EVS, at least in mice, relies on at least three distinct cholinergic mechanisms to modulate the resting discharge of vestibular afferents: (1) Activation of afferent muscarinic ACh receptors and subsequent closure of afferent KCNQ potassium channels, (2) activation of afferent $42 *$-containing nicotinic AChRs, and (3) sequential activation of 910 nicotinic AChRs and SK2 potassium channels in type II hair cells. In considering these data, a broader role for EVS function will be discussed in the context of the observed vestibular phenotypes in transgenic mice lacking these synaptic mechanisms, as well as including peripheral EVS mechanisms as potential targets for clinically-relevant anticholinergics.

Acknowledgements: This work was supported by NIH/NIDCD grant R01DC016974

\section{Additive integration of limb somatosensory inputs in the vestibular nuclei}

\section{(Abstract 43)}

Derek M. Miller, Ph.D.

Department of Otolaryngology, University of

Pittsburgh

Carey D. Balaban, Ph.D.

Departments of Otolaryngology, Neurobiology,

Communication Sciences and Disorders, and

Bioengineering, University of Pittsburgh

Andrew A. McCall, M.D.

Department of Otolaryngology, University of Pittsburgh

The central nervous system processes multiple sensory inputs, including vestibular and somatosensory signals, in order to maintain balance. We recently demonstrated that many neurons in the 
vestibular nuclei, site of the first central synapse of most vestibular afferents and a key component of the balance control circuit, receive convergent vestibular and limb somatosensory inputs. While it is established that neck proprioceptive inputs sum with and cancel vestibular inputs during head-on-body movements, it remains unclear if limb proprioceptive inputs that reach the vestibular nuclei are similarly encoded to permit additive integration with vestibular inputs. We sought to answer this question in the conscious cat model. Extracellular single unit recordings were obtained from 120 vestibular nucleus neurons during ramp-and-hold movements applied to the head in the roll plane (vestibular stimulation), to the hindlimb in the sagittal plane (limb somatosensory stimulation), and to both sites simultaneously. Neuronal responses were binned and fit with a nonlinear least squares regression model comprised of subcomponents of the applied stimuli. Model fits were excellent with mean R2 goodness of fit of 0.74 ( $\pm 0.17 \mathrm{SD}$; range $0.31-0.98$ ), thus confirming our hypothesis that the activity of vestibular nucleus neurons during combined vestibular and limb somatosensory activation is well-fit by an additive model. This finding suggests that convergent limb and vestibular inputs to vestibular nucleus neurons are not gated to allow only one input to predominate over the other. Rather, the inputs are combined allowing both to influence vestibular nucleus firing, presumably to govern vestibulospinal outflow.

Acknowledgements: Funding from NIH/NIDCD: K08-DC013571

\section{Changes in cardiovascular function and heart rate variability during infrared stimulation of vestibular endorgans}

\section{(Abstract 44)}

Darrian Rice, Department of Biomedical Engineering, University
of Miami

Giorgio P. Martinelli, Ph.D. and Gay R. Holstein, Ph.D.

Department of Neurology, Icahn School of

Medicine at Mount Sinai

Suhrud M. Rajguru, Ph.D.
Department of Biomedical Engineering, University of Miami and 3Department of Otolaryngology, University of Miami

Applications of sinusoidal galvanic vestibular stimulation and head tilts are known to evoke significant changes in the blood pressure (BP) and heart rate (HR) via the activation of the vestibulosympathetic reflex (VSR). To investigate the contribution of individual endorgans to this reflex, we have developed the application of pulsed infrared radiation (IR) to activate the posterior semicircular canal in a rodent model. Changes in BP and HR were studied in adult male Long-Evans rats weighing $300-500 \mathrm{~g}$, anesthetized with ketamine $(44 \mathrm{mg} / \mathrm{kg})$ and xylazine $(5 \mathrm{mg} / \mathrm{kg})$. A head post was cemented to the skull of each rat and attached to a customdesigned stereotaxic frame for restricted head movement during stimulation. Frequency modulated IR pulses $(1863 \mathrm{~nm}, 200 \mathrm{us}, 250 \mathrm{pps}$, varied radiant exposure) were delivered at $0.05 \mathrm{~Hz}$ to the posterior canal crista via a 200 or $400 \mu \mathrm{m}$ optical fiber. Mean $\mathrm{BP}$ and HR were measured via a small animal single pressure implantable device (DSI pressure sensing technologies, HD-S10) inserted into the femoral artery prior to stimulation. Eye movements simultaneously recorded using a custom-modified videooculography system (ISCAN Inc.) and postmortem micro computed-tomography confirmed the site of stimulation. In three rats, the vestibular system was activated with electrical stimulation (biphasic bipolar, $200 \mathrm{~Hz}$ pulse rate, $0.05 \mathrm{~Hz}$ modulation to mimic IR stimulation parameters) and results were compared to changes evoked by focused IR stimulation. Sinusoidal IR $(0.05 \mathrm{~Hz})$ delivered to the posterior canal induced an initial drop in both BP $(4.44 \pm 2.96 \mathrm{mmHg})$ and $\mathrm{HR}(17.45 \pm 13.07 \mathrm{bpm})$ followed by sinusoidal modulation. The responses ceased following IR stimulation. Corresponding eye movements and post-mortem microCT confirmed the posterior canal ampullary region to be the primary target of stimulation. Results of electrical activation of the peripheral vestibular system matched those from IR stimulation. In at least half of the animals, the high to low frequency ratio of heart rate variability (HRV) increased during IR stimulation over baseline resting condition. Posterior canal receptor activation evoked robust changes in $\mathrm{BP}$ and HR and the responses were confirmed with electrical stimulation. HRV analysis indicates sympathetic activation during posterior canal 
stimulation. Results are further suggestive of selective activation of the vestibular system by focused IR, which can be used to detail the VSR pathways and contributions of individual end organs.

Funding: NIH NIDCD 1R01DC008846 (GRH) and 1R01DC013798 (SMR)

\section{Vestibular control of blood pressure: neural mechanisms and role of cognition}

\section{(Abstract 45)}

Bill J. Yates, Ph.D., Derek M. Miller, Ph.D. And Andrew A. McCall, MD

Department of Otolaryngology, University of Pittsburgh School of Medicine, Pittsburgh, PA

Research using a variety of methods in both animal and human subjects over the past three decades demonstrated that inputs from the vestibular labyrinth contribute to adjusting blood pressure during movement and changes in posture (Yates et al, 2014). Lesion experiments showed that the rostral ventrolateral medulla (RVLM), an area of the reticular formation that regulates sympathetic nervous system activity, plays a key role in mediating vestibulo-sympathetic responses. Recent studies used a feline animal model to examine the processing of vestibular signals by RVLM neurons, which were identified based on their location and by having activity that was synchronized with changes in blood pressure during the cardiac cycle. In decerebrate cats, over half of RVLM neurons responded to $10-15^{\circ}$ tilts in vertical planes, while $1 \%$ of RVLM neurons responded to such rotations in conscious animals. However, in conscious cats the activity of $\sim 40 \%$ of RVLM neurons was modulated by $40^{\circ}$ tilts in the pitch plane; activity decreased in about half of these cells (by $-30 \pm 20 \%$, StDev) when the animal was positioned nose-up, while activity increased in the other half of the neurons during $40^{\circ}$ nose-up rotations (by $29 \pm 21 \%$ ). There was no apparent relationship between the change in neuronal activity and the change in heart rate during rotations, suggesting that the responses were not due to baroreceptor activation. Engagement of vestibulo-sympathetic responses during only large-amplitude tilts is physiologically relevant, since small-amplitude movements $(40 \%)$ do not produce peripheral blood pooling that necessitates an increase in sympathetic nervous system activity. Thus, our data suggest that vestibular signals elicit rapid increases in sympathetic nervous system activity during movements that are large enough to produce orthostatic hypotension, and that supraspinal mechanisms (eliminated by decerebration) are critical in adjusting the sensitivity of the response so it is only engaged by large movements. When tilts were cued by a light signal, no alterations in RVLM neuronal activity occurred prior to the tilt, showing that anticipation of movement does not cause a feedforward increase in sympathetic nervous system activity; the responses only occur as the movement progresses.

\section{References}

1. Yates et al. (2014) Compr. Physiol. 4:851-887.

Acknowledgements: Funded by NIH/NIDCD R01DC013788.

\section{Size matters for vestibular perception but not vestibular reflexes}

\section{(Abstract 46, Poster Location A)}

Eric Anson, PT, $\mathrm{PhD}^{1,2}$, Yoav Gimmon, PT, $\mathrm{PhD}^{2}$, Edward Chen ${ }^{3}$, Peter Boutros, $\mathrm{BS}^{3}$, Michael

Schubert, $\mathrm{PT}, \mathrm{PhD}^{2}$

${ }^{1}$ Department of Otolaryngology, University of Rochester, Rochester, NY, USA

${ }^{2}$ Department of Otolaryngology - Head \& Neck Surgery, Johns Hopkins University School of Medicine, Baltimore, MD, USA

${ }^{3}$ Department of Biomedical Engineering, University of Rochester, Rochester, NY, USA

${ }^{4}$ Department of Biomedical Engineering, Johns Hopkins University, Baltimore, MD, USA

Background: Chronic dizziness can lead to abnormal self-motion perception. Perceptual training may improve chronic dizziness. Prior studies limited the number of rotations to avoid attenuation of both rotational perception and gain of the vestibulo-ocular reflex (VOR), as occurs with repeat constant velocity rotations. We hypothesize that neither accuracy in perception of self-motion nor VOR gain will degrade with repeat step rotations, but that perceptual accuracy of rotational magnitude depends on rotation size. 
Methods: 15 healthy adults, mean age 31.2 (SD = 7.96) years sat in a motorized rotary chair and experienced 100 velocity trapezoid (peak velocity of 60 degrees/second) position step rotations (10 blocks of 10 rotations) of $45,90,135$, and 180 degrees in the dark while VOR gain was measured. Rotation directions alternated right and left and rotation size was randomized for each subject. After each rotation, subjects verbally reported the perceived rotation size. Perceptual responses were compared to actual rotation size to determine accuracy. Repeated measures ANOVAs were used to determine if 1) perceptual accuracy or VOR gain changed with rotation size; and 2) average perceptual accuracy or VOR gain decreased across blocks. Alpha was set to 0.05 .

Results: Perceptual accuracy for vestibular spatial orientation significantly declined with increasing rotation amplitude $(\mathrm{F}(3,59)=18.22, \mathrm{p}$ 0.001). Average perceptual accuracy was stable across all blocks $(F(9,149)=1.52, \mathrm{p} 0.208)$. VOR gain was stable across all blocks $(\mathrm{F}(9,108)=2.11, \mathrm{p} 0.117)$, but was significantly smaller for 45 degree rotations $(F(3,51)=9.10, p 0.001)$.

Conclusions: These results indicate that vestibular spatial orientation perception is less accurate as rotation size increases, while VOR gain is reduced for smaller rotations due to lower peak velocity. The number of position step rotations does not impact perceptual accuracy or VOR gain across 10 blocks. This provides the groundwork for developing novel treatments for vestibular perceptual problems.

\section{References}

1. Clément G, Tilikete C, Courjon J-H (2008) Retention of habituation of vestibulo-ocular reflex and sensation of rotation in humans. Exp Brain Res 190:307-315.

2. Cohen BS, Provasi J, Leboucher P, Israël I (2017) Effects of vestibular disorders on vestibular reflex and imagery. Exp Brain Res 235:2181-2188.

Funding source: Supported in part by NIDCD T32 DC000023 (E Anson).

\section{Focused ultrasound stimulation can preferentially activate vestibular organs}

\section{(Abstract 47)}

\author{
MM Iversen, BR Pope, RD Rabbitt \\ University of Utah, Salt Lake City, UT
}

Introduction: Neural encoding of gravito-inertial acceleration by inner ear vestibular organs primarily uses action potential firing rate modulation (rate code) to transmit traditional low frequency vestibular information [1], and action potential timing (phase locking) to transmit high frequency sound, acceleration, and vibration [2]. We recently demonstrated that low intensity focused ultrasound (LiFU) applied to the semicircular canal crista, utricular macula or saccular macula can activate vestibular afferent neurons [3]. Phase-locked responses can be preferentially evoked in transienttype afferent neurons and modulation of firing rate in sustained-type afferent neurons. Results have relevance to applications of $\mathrm{LiFU}$ to remote vestibular stimulation, as well as quantifying coding of high frequency stimuli by vestibular organs.

Methods: Focused $5 \mathrm{MHz}$ ultrasound was applied to the vestibular organs in both pulsed discrete packets with rates of 10-5000 Hz and continuous sinusoidal modulation with rates of $0.5-10 \mathrm{~Hz}$. Single-unit afferent neurons were recorded and analyzed for phase-locking strength (vector strength) and timing (stimulus-triggered histograms) across a range of ultrasound amplitudes. Background discharge statistics were recorded to examine correlates with temporal coding properties of individual organs.

Results: Vestibular afferent neurons with irregular interspike intervals at rest, responded to pulsed ultrasound with high vector strength to ultrasound pulses. Vestibular afferent neurons with regular interspike intervals at rest, responded to continuous ultrasound by modulating firing rate. Afferents innervating the otolith organs responded with higher strength than afferents innervating cristae. Responses in otolith organs mimic those elicited by high frequency vibrational acceleration.

Conclusion: Vestibular end organ sensitivity to LiFU is the result of the acoustic radiation force acting to deflect sensory hair bundles as shown 
previously [3]. Responses are stronger in the otolith organs due to the acoustic radiation force generated by the high impedance mismatch between the otolithic mass and surrounding tissues. LiFU can be used to preferentially activate specific vestibular organs and neurons types.

\section{References}

1. SG Sadeghi, J Neurosci (2007)

2. IS Curthoys, Brain Res Bull (2012)

3. MM Iversen, J Acoust Soc Am (2017)

Funding: NIDCD R01 DC006685 \& R21 DC016443

\section{Adaptation of vestibular heading perception using visual offsets}

\section{(Abstract 48, Poster Location C)}

Raul Rodriguez, M.S. and Benjamin T. Crane, MD, Ph.D.

\section{University of Rochester, Rochester, NY}

Persistent dizziness symptoms often occur in patients that have undergone unilateral vestibular lesions. Rehabilitation addresses many of the perceptual problems, but previous work has shown that these patients have persisting biases in heading perception. This ongoing perceptual bias may be the source of symptoms. Developing an adaptation technique that addresses these heading biases could help in dealing with long-term vestibular symptoms. This study explores heading adaptation in normal subjects. Previous experiments in visual-vestibular adaptation focused on cue reliability and kept the angular discrepancy between visual and inertial cues between 2 and $10^{\circ}$ [1]. We aim to explore the boundaries of vestibular adaptation with greater angular discrepancies and eventually expand the subject pool to patients that have had a unilateral vestibular lesion. Subjects were asked to report inertial heading direction using a forced choice task in which they reported headings as left or right of midline, until the point of subjective equality (PSE) was reached (as previously published) to establish a baseline heading perception. The subjects were then exposed to an inertial stimulus and a concurrent visual stimulus. The visual stimulus was offset by $+/-20^{\circ}$ (only one offset, + or -, per trial). Subjects were still asked to report inertial heading direction.
The inertial heading perception PSE was found to be shifted in the direction of the visual offset. The subjects completed two full trials (approx. 10 minutes each) with the same visual offset. They were then asked to report inertial heading direction without a visual cue. In 5 subjects that have each completed 2 sessions of leftward adaptation, baseline inertial heading perception had a PSE of $1.9 \pm 5.5^{\circ}$ $($ mean $\pm \mathrm{SD})$. After exposure to two trials with a $-20^{\circ}$ visual offset. We found a shift of $4.5 \pm 4.2^{\circ}$ (p0.05 two-tailed t-test) in the direction of the visual stimulus. Exposure to an offset visual stimulus can change the perception of inertial stimuli. This raises the possibility that applying an adaptation to patients with long-term unilateral vestibular lesions could help address persistent perceptual errors which could be the source of residual symptoms.

\section{References}

1. Zaidel, A., A.H. Turner, and D.E. Angelaki, Multisensory Calibration Is Independent of Cue Reliability. The Journal of Neuroscience, 2011. 31(39): p. 13949-13962.

Acknowledgements: Funded by R01-DC013580

\section{Variability in vestibular thresholds at multiple frequencies across populations}

\section{(Abstract 49, Poster Location D)}

Farzad Ehtemam

The Ohio State University

Susan King, Adrian Priesol, Richard F. Lewis

Jenks Vestibular Physiology Lab, MEEI; Harvard Medical School Boston MA

Daniel M. Merfeld

The Ohio State University and Naval Medical Research Unit - Dayton $\mathrm{OH}$

Vestibular thresholds are reliable measures in the assessment of the vestibular function as well as being indicators of balance deficits across populations [1],[2]. Vestibular thresholds have been measured at many different frequencies [3], [4]. Although vestibular organs respond to stimuli over a wide range $(0.01-20 \mathrm{~Hz})$ [5], 
the range of perceptual threshold responses is more limited and affected by various factors. For instance, pathological factors can reduce thresholds [2] and increases in threshold have been documented in geriatric populations [1]. Due to physiological and cognitive factors, responses at certain frequencies may provide a more coherent assessment of the vestibular function. However, this effect of frequency on variability and consistency of vestibular thresholds across subjects is not well understood. Here we investigated the betweensubject variability of roll tilt thresholds in migraine and vestibular migraine patients as well as healthy controls. All groups were tested at four frequencies $(0.05,0.1,0.20 .5 \mathrm{~Hz})$ in a randomized design. A forced-choice, oneinterval, direction discrimination task was used. The data showed at least one subject's data as an outlier at all frequencies except $0.2 \mathrm{~Hz}$ for which no outliers were observed in any of the subject groups. The thresholds at $0.05 \mathrm{~Hz}$ showed at least one outlier for each group. To better understand how the threshold data compared between different populations we looked at the distribution of samples across frequencies. It is well documented that thresholds follow a lognormal distribution [1],[6]. We observed the distributions at $0.2 \mathrm{~Hz}$ to be closer to log normal for the vestibular migraine group. These results show higher consistency and lower variability at $0.2 \mathrm{~Hz}$ compared to $0.05 \mathrm{~Hz}$. Although the underlying mechanisms responsible for these differences have yet to be determined, these results make sense intuitively. One possible source of these differences can be lack of adequate attention during trials at $0.05 \mathrm{~Hz}$ since these trials are four times longer than $0.2 \mathrm{~Hz}$ trials. This can potentially result in lapses in subjects which in turn increases the variability and can result in outliers. Further research and more comparisons are required to corroborate these preliminary results and draw final conclusions.

\section{References}

1. Bermúdez Rey et al, Front Neurol, 2016

2. Lewis et al, J Vestib Res, 2011
3. Valko et al, J. Neurosci., 2012

4. Grabherr et al, Exp Brain Res, 2008

5. Rabbitt et al, in The Vestibular System, 2004

6. Benson et al, Aviat Space Environ Med, 1989.

Acknowledgments: Funded by NIH/NIDCD R01DC014924 and R01-DC012528

\section{Frontal scalp potentials foretell perceptual choice confidence}

\section{(Abstract 50, Poster Location F)}

Koeun Lim ${ }^{1,2}$, Wei Wang ${ }^{3,4}$, Daniel M. Merfeld ${ }^{1,2,5,6}$

${ }^{1}$ Jenks Vestibular Physiology Lab, Massachusetts Eye and Ear Infirmary - Boston MA

${ }^{2}$ Program in Speech and Hearing Bioscience and Technology, MIT-Harvard Division of Health Sciences and Technology, Massachusetts Institute of Technology Cambridge MA

${ }^{3}$ Department of Medicine and Neurology, Brigham and Women's Hospital - Boston MA ${ }^{4}$ Department of Medicine, Harvard Medical School - Boston MA

${ }^{5}$ Department of Otolaryngology, Harvard Medical School - Boston MA

${ }^{6}$ The Ohio State University, Columbus OH and Naval Medical Research Unit - Dayton $\mathrm{OH}$

When making decisions, people naturally ask two implicit questions: how soon can I make a decision, and how certain am I? In perception, people's confidence (how certain?) shows a non-monotonic relationship with response-time (how soon?), such that choice confidence can either increase or decrease with response-time. While a fronto-parietal network has been implicated as a neural substrate that binds choice confidence and action (e.g., response-time), the dynamic interplay between choice behaviors within such a network has not been clarified. Here we show that choice confidence contributes to frontal event-related potentials (ERPs) prior to a decision. 15 healthy human participants viewed a stationary Gabor image randomly oriented at one of four tilt angles, and their task was to report perceived 
orientation (clockwise or counterclockwise) upon reaching a decision without rushing by pressing a button. Stimulus turned off when a button was pressed, and then participants verbally reported choice confidence between 50\% (random guess) and $100 \%$. This task yielded three behavioral measurements, response-time, binary choice accuracy, and choice confidence. We simultaneously measured scalp potentials with a 64-channel BrainVision EEG system while participants performed choice tasks. Our primary novel finding was that the second positive peak (P2) of frontal ERP between $400 \mathrm{~ms}$ and $600 \mathrm{~ms}$ from the stimulus onset reflects and most strongly correlated with choice confidence (P2 curvature, F3,42=7.58, $\mathrm{p}=0.0004)$ than response-time or stimulus level $(\mathrm{z} \geq 8.82, \mathrm{p} 0.0001)$. On the other hand, centroparietal ERP was most strongly correlated with responsetime ( $\mathrm{z} \geq 9.05, \mathrm{p} 0.0001)$, consistent with the current theory suggesting that activity in parietal areas reflect choice action. The novel frontal ERP component (400 600ms) occurs before the response (mean response-time $864 \mathrm{~ms}$ ), which helps explain the inverse relationship between choice confidence and response-time (i.e., higher confidence for shorter response-time) when choice accuracy is emphasized over speed. Our findings provide the first neural representation showing that confidence may take a causal role in the current decision-making task - "I decided earlier because I am confident," - consistent with a dual-route model of metacognition.

\section{Differences in compensatory saccades metrics for ipsilesional vs contralesional head impulses in unilateral vestibular deafferentation}

\section{(Abstract 51, Poster Location H)}

\section{Andrew R. Wagner, P.T., D.P.T., N.C.S. Northeast Ohio VA Medical Center, Cleveland, $\mathrm{OH}$}

Michael C. Schubert, P.T., Ph.D.

Johns Hopkins University, Baltimore, MD

Video head impulse testing (vHIT) is useful to document the extent of peripheral vestibular loss by comparing vestibuloocular reflex gain (VOR, eye/ head velocity) between the lesioned and contralesional labyrinths (Halmagyi, et al. 2017). Our objective was to extend this assessment by comparing compensatory saccade (CS, saccades that occur in the direction of the deficient VOR) metrics during ipsilesional and contralesional vHIT in patients who have undergone unilateral vestibular deafferentation (UVD). Yaw vHIT (ICS Otometrics, Taastrup, Denmark) was completed in 40 individuals post UVD. To date, 15 subjects have been analyzed using a custom MatlabTM (Natick, MA) program to determine the peak velocity, latency, and frequency of CS (overt and covert) for both ipsilesional and contralesional impulses. A repeated measures MANOVA was used to identify the effect of impulse direction on CS metrics using SPSSTM (IBM Corp. Armonk, NY). In order to determine the difference in individual saccade metrics between ipsi and contralesional vHIT we applied pairwise comparison of means using a paired t-test with Bonferroni correction. Pearson correlation coefficient (with 95\% CI) was used to assess the correlation between CS metrics and VOR gain. The overall model was significant comparing CS metrics between ipsi and contralesional head rotations $(\mathrm{F}=19.034, \mathrm{p} .001)$. Our data analysis support that CS are present during contralesional head rotation, but at velocities (Ipsi: $73.03 \pm 43.15 \mathrm{deg} / \mathrm{sec}$; Contra: $31.04 \pm 20.28 \mathrm{deg} /$ sec, $\mathrm{p}=.002$ ) and frequencies (Ipsi: $2.31 \pm 0.67$ saccades/impulse; Contra: $1.36 \pm 0.54$ saccades/ impulse, $p$.001) less than their ipsilesional counterpart. A lower ipsilesional gain was associated with the recruitment of higher peak velocities of CS during ipsilesional vHIT $(\mathrm{r}=-0.595 ; 95 \% \mathrm{CI},-0.849$ to $-0.119, \mathrm{p}=.019)$. CS latency however was similar for ipsi and contralesional vHIT (Ipsilesional: $153.19 \pm 19.65 \mathrm{~ms}$; Contralesional: $166.82 \pm 56.35$ $\mathrm{ms}, \mathrm{p}=0.370)$ and showed an inverse relationship with ipsilesional VOR gain $(\mathrm{r}=-0.548 ; 95 \% \mathrm{CI}$, -0.828 to $-0.049, \mathrm{p}=.035)$ suggesting that latency may have a limited role in compensating for a low VOR gain. The number of CS recruited during contralesional vHIT appear to be related to the extent of vestibular loss on the lesioned side $(\mathrm{r}=0.529$; $95 \% \mathrm{CI}, 0.023$ to $0.819, \mathrm{p}=.043$ ); additional work will determine if contralesional CS have a functionally relevant role in gaze stability post UVD.

\section{References}

1. Halmagyi GM, Chen L, MacDougall HG, Weber KP, McGarvie LA, Curthoys IS. The Video Head Impulse Test. Frontiers in Neurology. 2017. 8:258. 
Acknowledgements: Department of Defense Award W81XWH-15-1-0442

\section{Functional impact of diverse sodium currents on vestibular afferent neuron firing patterns}

\section{(Abstract 52, Poster Location I)}

Selina Baeza Loya, Ruth Anne Eatock, Ph.D. Neurobiology, University of Chicago, Chicago IL

An outstanding issue in neural coding is the impact of diverse ionic currents on the transmission of sensory stimuli information by neurons. The vestibular system transmits sensory information with two populations of vestibular afferent neurons (VAN), which differ in the regularity of timing of action potentials (AP). The two kinds of AP timing are specialized for different encoding strategies (rate and temporal)1, which are optimized for different kinds of sensory information. We ask how the two sensory encoding methods arise from differences in the voltage-dependent sodium $(\mathrm{NaV})$ currents that initiate APs. Recent work from our lab indicated expression of all $\mathrm{Na}$ channel proteins in VAN2, which differ in key properties affecting their voltage dependence and time course, and may carry currents of different modes (i.e., transient, persistent, resurgent). I seek to determine how diverse $\mathrm{NaV}$ currents influence the shape and timing of APs. Using whole-cell patch-clamp recordings, I discovered that some neurons expressed persistent $\mathrm{NaV}$ current elicited with slow voltage ramps and that other neurons express resurgent $\mathrm{NaV}$ current. I hypothesize that these non-inactivating modes repolarize VAN after each spike, promoting the sustained firing that distinguishes the VAN population that fires with regular timing. My longterm goal is to explain how biophysical properties have evolved to analyze sensory stimuli into key features.

\section{References}

1. Jamali M., et al, (2017). Nature Communications 7(13229),

2. Liu X.P., et al, (2016). Journal of Neurophysiology 115(2536).

\section{Prediction of functional limitations in balance after tests of tandem walking and standing balance}

\section{(Abstract 53, Poster Location O)}

Helen S Cohen, $\mathrm{PhD}$

Dept of Otolaryngology, Baylor College of Medicine

Susan P Williams, MD

Dept of Medicine, Baylor College of Medicine

Haleh Sangi-Haghpeykar, $\mathrm{PhD}$

Dept of Obstetrics and Gynecology, Baylor College of Medicine

Ajijtkumar P Mulavara, $\mathrm{PhD}$

KBRwyle, Houston Jacob J Bloomberg , $\mathrm{PhD}$, NASA/ Johnson Space Center

Geriatricians have no good tests with which to predict decreased balance skill in aging patients. Subjects with no apparent balance problems were tested in the Geriatric Medicine clinic on Tandem Walking (eyes closed) and three conditions of Romberg on foam with eyes closed: head stationary, head moving in yaw, head moving in pitch, and retested 1.5 to 2 years later. The hypothesis was that some pre-test results would predict subsequent functional balance or reports of dizziness or vertigo. The analyses showed slight but significant decrements in Romberg tests over time, but no relationship to reports of falls, vertigo, lightheadedness or use of a cane/ hip surgery. Tandem walking did not change significantly over time but was moderately related to use of a cane/ having had hip surgery. It was not related to vertigo or lightheadedness. These data suggest that tandem walking results may help the physician to determine which patients are most at risk for clinically significant changes in their gait status.

Supported by NIH grant 2R01 DC009031. 


\section{An integrative and multisensory approach to care for a service member with a complicated vestibular}

\section{(Abstract 54, Poster Location P)}

\author{
History: A Case Report \\ Marcy Manes Pape, PT, DPT \\ National Intrepid Center of Excellence, Walter \\ Reed National Military Medical Center
}

\begin{abstract}
Background and purpose: Despite well-established interventions to address individual vestibular diagnoses, there are no protocols for multisensory interventions addressing simultaneous central and peripheral vestibulopathy, especially when treatment is confounded by variables such as hearing, vision, pain, and cognitive challenges. Complex vestibular cases often have segmented care, and no studies found examine the importance of multidisciplinary vestibular evaluations with integrated multisensory treatment. This study highlights one service member whose success improved with an integrative treatment program.
\end{abstract}

Case description: A 45-year-old service member with central and peripheral vestibulopathy, including superior canal dehiscence, Meniere's disease, cochlear implantation, migraines, visual challenges, anxiety from chronic dizziness and cervicogenic involvement, undergoing multisensory evaluations and treatments during his course of gentamycin injections.

Results: Multidisciplinary care improved diagnostic capabilities, and a multisensory rehabilitation approach over 3 months improved objective balance scores and self-impression of balance and functional activities.

Discussion: With a complicated vestibular case, consider an integrative multisensory approach to care for successful outcomes.

\section{References}

1. Fausti, S. A., Wilmington, D. J., Gallun, F. J., Myers, P. J., \& Henry, J. A. (2009). Auditory and vestibular dysfunction associated with blast-related traumatic brain injury. Journal of Rehabilitation Research \& Development, 46(6).
2. Pogoda, T. K., Hendricks, A. M., Iverson, K. M., Stolzmann, K. L., Krengel, M. H., Baker, E., \& Lew, H. L. (2012). Multisensory impairment reported by veterans with and without mild traumatic brain injury history. Journal of Rehabilitation Research and Development, 49(7), 971.

3. Herdman, S. J., Hall, C. D., \& Delaune, W. (2012). Variables associated with outcome in patients with unilateral vestibular hypofunction. Neurorehabilitation and Neural Repair, 26(2), 151162.

\section{The effect of postural instability on simulator seasickness and performance in a shooting task}

\section{(Abstract 55, Poster Location Q)}

Kyle A. Pettijohn, Ph.D.

The Henry M. Jackson Foundation for the Advancement of Military Medicine, Bethesda, MD and Naval Medical Research Unit-Dayton, $\mathrm{OH}$

Dominick V. Pistone

Oak Ridge Institute for Science and Education, Oak Ridge, TN and Naval Medical Research Unit Dayton, $\mathrm{OH}$

Andrew L. Warner B.C.J., Grant J. Roush B.A. The Henry M. Jackson Foundation for the Advancement of Military Medicine, Bethesda, MD and Naval Medical Research Unit-Dayton, $\mathrm{OH}$

LT Adam T. Biggs Ph.D., MSC, USN

Naval Medical Research Unit-Dayton, $\mathrm{OH}$

Motion sickness is a problem for many people; however, it is especially pressing for military personnel who need to operate in moving environments. The current study investigated the underlying cause of motion sickness by testing one of the leading theories, postural instability theory (Riccio \& Stoffregen, 1991). Subjects experienced motion profiles while they performed a virtual reality shooting task and reported the severity of their symptoms. There were two conditions in which postural instability was manipulated. In one, subjects could adapt their posture to the motion profile (Free), and in the second, they were prevented from doing this by having their feet fixed in place on the moving 
platform (Fixed). Subjects completed both conditions to control for individual differences in motion sickness susceptibility. Postural stability was assessed quantitatively by measuring sample entropy and ellipsis area of the head. There were no differences in sickness symptoms between the conditions, but there was reduced postural stability in the Fixed condition. Performance on the shooting task was greatly affected by motion, with a reduction in accuracy of approximately $40 \%$. The results do not support postural instability as the primary causal factor in motion sickness and demonstrate the importance of accounting for motion when conducting training.

\section{Reference}

1. Riccio \& Stoffregen, (1991). Ecological Psychology 3(3).

Acknowledgement: Funded by the Office of Naval Research under work unit number

\section{Morphometric analysis of otoconia in a mouse model of pendred syndrome by x-ray computed microtomography.}

\section{(Abstract 56, Poster Location R)}

Taku Ito, MD.; Ph.D., Takamori Takeda, MD. Keiji Honda, MD.; Ph.D., Yoshiyuki Kawashima, MD.; Ph.D. Takeshi Tsutsumi, MD.; Ph.D.

Department of Otorhinolaryngology, Tokyo Medical and Dental University, Tokyo, Japan

Hypothesis: A three-dimensional reconstruction of otoconia by microCT may be useful for elucidation of an etiology for equilibrium disturbance in a mouse model of Pendred syndrome.

Background: Vestibular dysfunction in Pendred syndrome is an inconsistent and variable. Slc26a4-/mice also manifest variable degrees of vestibular dysfunction as evidenced by gait unsteadiness, circling behavior, and head tilting. Scanning electron microscopic studies of vestibular end organs demonstrated the abnormally enlarged otoconia after 7 days of age.

Methods: The otic capsules of $\operatorname{Slc} 26 \mathrm{a} 4 \Delta /+$ and $\operatorname{Slc} 26 \mathrm{a} 4 \Delta / \Delta$ mice at 8 days and 1 and 3 months of age were imaged with micro CT, inspeXio SMX$100 \mathrm{CT}$, at 10-micron resolution. A multiplanar reconstructed view was used to calculate the width of saccule, utricle and semicircular canals and delineate the shape, size and location of otoconia.

Results: The ossicles, osseous labyrinth and otoconia were clearly visualized by microCT. $\operatorname{Slc} 26 \mathrm{a} 4 \Delta / \Delta$ mice demonstrated enlarged saccule and utricle but almost normal semicircular canals. The Slc26a $4 \Delta /+$ mice showed linearly distributed otoconia in the utricle and saccule. In the utricle of Slc26a $4 \Delta / \Delta$ mice, nearly 10 giant otoconia were discretely distributed at P8. The number of otoconia decreased with age. In the sacclue, otoconia were fewer or absent than those in utricle at P8. No ectopic otoconia were observed in any semicircular canals.

Conclusions: We succeeded in non-destructively delineating the giant otoconia of $\operatorname{Slc} 26 \mathrm{a} 4 \Delta / \Delta$ mice under conditions with extremely few artifacts by microCT. These results are expected to be useful for elucidation of the etiology for equilibrium disorder in Pendred syndrome.

\section{Quantifying the effect of chronic vestibular impairment on behavoiral responses to dynamic visual motion}

\section{(Abstract 57, Poster Location V)}

\author{
Ashley C. Zaleski-King ${ }^{1}{ }^{2}$, Robin L. Pinto ${ }^{1}$, and \\ Douglas S. Brungart ${ }^{1}$ \\ ${ }^{1}$ Walter Reed National Military Medical Center, \\ Bethesda, Maryland \\ ${ }^{2}$ Gallaudet University
}

After vestibular insult, a subset of patients continues to report dizziness symptoms that persist beyond the acute stage and into a chronic stage. This persistence of symptoms is associated with visual motion sensitivity (VMS) (Cousins et al, 2014). Chronically dizzy patients with VMS can be difficult to manage because objective clinical vestibular findings may not fully explain the continuation of their symptoms and the persistance of functional deficits in complex visual environments (Guerraz et al, 2001). The purpose of this study was to provide empirical data describing the relationship between vestibular impairment, self-reported visual motion sensitivity 
and the effect of dynamic visual information on visual perception of verticality, postural stability, and oculomotor control in adults with chronic dizziness symptoms. Postural stability (sway path length) and subjective visual vertical (SVV) perception were measured in a dynamic visual environment presented through a head-mounted display). The visual stimulus was developed at WRNMMC using the Oculus Rift Development Kit 2, v.0.5.0 in the Unity 3D development environment and the $\mathrm{CH}$ programming language. Bivariate regression analyses were performed in 50 adult subjects to determine if the behavioral responses to dynamic visual motion were associated with clinically identifiable vestibular impairment and self-reported measures of visual motion sensitivity (visual vertigo analog scale), self-reported handicap (Dizziness Handicap Inventory) and self-reported levels of anxiety (Generalized Anxiety Disorder-7). We report: (a) a strong age effect in the weighting of dynamic visual cues used for SVV perception, (b) that level of self-reported anxiety was associated with increased sway path length in the absence of earth-fixed reference frames, and (c) that clinically identifiable vestibular impairment did not distinguish performance on any self-reported or behavioral response to visual motion. These early findings may have important implications for identifying patients who might benefit from vestibular rehabilitation therapy focused on habituation to visual motion.

\section{References}

1. Cousins, S., Cutfield, N. J., Kaski, D., Palla, A., Seemungal, B. M., Golding, J. F., ... \& Bronstein, A. M. (2014). Visual dependency and dizziness after vestibular neuritis. PloSone, 9(9), e105426

2. Guerraz, M., Yardley, L., Bertholon, P., Pollak, L., Rudge, P., Gresty, M. A., \& Bronstein, A. M. (2001). Visual vertigo: symptom assessment, spatial orientation and postural control. Brain, 124(8), 1646-1656.

\section{Afferent modulation by excitatory GABAergic efferent inputs}

\section{(Abstract 58, Poster Location W)}

Yugandhar Ramakrishna ${ }^{1}$, Vishal Raghu ${ }^{1,2}$ and Soroush G. Sadeghi ${ }^{1,2}$

${ }^{1}$ Center for Hearing and Deafness, Dept. of

Communicative Disorders and Sciences, State
University of New York at Buffalo, Buffalo, NY

${ }^{2}$ Neuroscience Program, State University of New York at Buffalo, Buffalo, NY

The vestibular efferent fibers project bilaterally to innervate bouton and calyx afferent terminals and type II hair cells. Multiple neurotransmitters have been identified in the vestibular efferent fibers, including acetylcholine (ACh) and gamma amino butyric acid (GABA). Using in vitro patch clamp recordings we show that membrane properties of calyx afferent terminals were modulated by baclofen, a metabotropic GABA-B agonist. Through inhibition of voltage sensitive calcium channels and consequent inhibition of calcium sensitive potassium channels, baclofen exerted an excitatory effect on calyces, resulting in lower action potential thresholds (i.e., higher sensitivity) and faster responses to step depolarizations. Consistent with these findings, in vivo intracochlear injection of baclofen produced an increase in synchronized response of irregular fibers innervating the otolith endorgans as measured by vestibular sensory evoked potentials (VsEP, P1N1 amplitude) and a decrease in P1 latency. Intracochlear injection of a GABA-B antagonist (CGP 35348) had the opposite effect on VsEP responses. Together, these results provide evidence for an excitatory GABAergic efferent-mediated effect on vestibular afferents. These effects are complementary to excitatory cholinergic efferent-mediated effects through muscarinic ACh receptors and inhibition of KCNQ channels. We propose that efferent inputs play an important role in adjusting the resting discharge and response properties of irregular afferents and altering the tradeoff between tonic (regular) and phasic (irregular) peripheral pathways.

These studies were funded by NIH-NIDCD grant 5R03DC015091 and a research grant from the American Otological Society (AOS).

\section{Rebalancing the vestibular system by unilateral rotations}

\section{(Abstract 59, Poster Location X)}

Navid G. Sadeghi ${ }^{1}$, Bardia Sabetazad ${ }^{2}$, Nayer

Rassaian ${ }^{1}$, Soroush G. Sadeghi ${ }^{3,4}$

${ }^{1}$ Dept. of Physiology, Shaheed Beheshti University of Medical Sciences and Health Services, Tehran, Iran 
${ }^{2}$ Audiology and Dizziness Center, Day General Hospital, Tehran, Iran

${ }^{3}$ Center for Hearing and Deafness and 4 Neuroscience Program, State University of New York at Buffalo, Buffalo, NY

Natural compensation following unilateral vestibular lesions does not use the full adaptation capacity of the system. For example, it has been shown that the vestibulo-ocular reflex (VOR) could be further improved by using rehabilitation exercises and visual - vestibular mismatch paradigms. We hypothesized that rotation of patients with asymmetric vestibular function towards the weaker side could result in an increase in the response of neurons to rotations in that direction, pushing the system to rebalance the activity on the two sides. Sixteen subjects with VOR asymmetry quantified by an abnormal directional preponderance (DP) were exposed to a unidirectional rotational paradigm, which consisted of five trapezoid unidirectional rotations $(320 \mathrm{o} / \mathrm{s})$ toward the weaker side. Care was taken to slowly stop the rotation in order to avoid stimulation in the opposite direction during deceleration. To study the short-term effect, VOR responses were measured before and 10,40, and 70 min after a single unidirectional rotational rehabilitation session. To investigate long-term effects, the VOR gain was measured before and 70 min after rehabilitation in 6 separate sessions over a one month period. We found a significant decrease in VOR asymmetry in both short-term and long-term groups. Even 10 min after a single session there was a decrease in DP and asymmetry, which reached normal values by the second session in most cases. Our results show that unidirectional rotations could provide a supervised rehabilitation method through a purely vestibular stimulus that could effectively improve the asymmetry even in patients with compensated vestibular dysfunction.

This study was funded by NIH-NIDCD grant 5R03DC015091 and a research grant from the American Otological Society (AOS).

\section{Reliability of bone conduction vestibular evoked myogenic potentials in children and adolescents}

\section{(Abstract 60, Poster Location Y)}

Nicole Greenwalt

\author{
BS-Third year AuD student at The Ohio State \\ University. \\ Jessie Patterson
}

PhD-Senior Audiology Research Associate at Boys

Town National Research Hospital (BTNRH).

Amanda Rodriguez PhD-Post-doctoral Fellow at BTNRH, Assistant Professor in the Department of Special Education and Communication Disorders at the University of Nebraska-Lincoln.

Denis Fitzpatrick, PhD-Senior Research Developer and Coordinator of Research Engineering at BTNRH.

Kristen Janky, PhD- Director of the Vestibular and Balance Laboratory at BTNRH.

Recent research has supported the use of Vestibular Evoked Myogenic Potential (VEMP) for assessing vestibular function in children (1-3). Bone conduction vibration (BCV) stimuli for VEMP testing are clinically desirable for multiple reasons. However, no accepted standard exists for stimulus type and the reliability of BCV devices has not been investigated in children. The objective of the current study was to determine (a) the optimal BCV VEMP method by measuring response rates and reliability in a group of 22 adolescents and children and (b) if estimations of bone density predict the efficiency of $\mathrm{BCV}$ stimulation of the otolith organs. When comparing the reliability of the B-71, reflex hammer, and Mini-shaker, analyses indicate that the optimal method varies by age and VEMP type. In addition, wrist circumference and BMI were negatively correlated with both cervical VEMP and ocular VEMP amplitudes in response to all BCV methods within the child, adolescent, and adult groups, suggesting estimations of bone density can be used to predict BCV efficiency. Further research is needed to determine the appropriate role for predictors of bone density in determining VEMP stimulus level and BCV stimulation type.

\section{References}

1. Wang S., Hsieh W., Young Y. (2013). Development of ocular vestibular-evoked myogenic potentials in small children. Laryngoscope, 123(2): 512-517. 
2. Zhou G., Dargie J., Dornan B., Whittemore K. (2014). Clinical uses of cervical vestibular-evoked myogenic potential testing in pediatric patients. Medicine, 93(4): 1-5.

3. Chou, C., Hsu, W., Young, Y. (2012). Ocular vestibular-evoked myogenic potentials via bone conducted vibration in children. Clinical Neurophysiology, 123: 1880-1885.

Acknowledgements: This work was supported by the National Institute of General Medical Sciences of the National Institutes of Health under award number P20GM109023 and 5T32DC00013-36 and by the National Institute on Deafness and Other Communication Disorders under award numbers T35 DC 008757 and R03DC015318.

\section{Pharmacological characterization of synaptic mechanisms governing the responses of mammalian vestibular afferents to efferent stimulation.}

\section{(Abstract 61, Poster Location Z)}

Todd Schneider MD, Choongheon Lee PhD, Allison Morningstar, Bao Dang, J. Chris Holt, PhD University of Rochester, Rochester, NY

Electrical stimulation of VENs in mammals excites primary vestibular afferents along two distinct time scales suggesting that activation of multiple postsynaptic mechanisms is involved. While activation of acetylcholine $(\mathrm{ACh})$ receptors accounts for EVS actions in other vertebrate vestibular preparations, synaptic mechanisms underlying afferent responses to EVS stimulation in mammals are unresolved. In this work, we sought to determine if the release of $\mathrm{ACh}$ and subsequent activation of $\mathrm{ACh}$ receptors do in fact account for efferentmediated afferent excitation in mammals. To characterize EVS actions, we recorded spontaneous afferent activity from the superior vestibular nerve of anesthetized mice while stimulating VENs in the brainstem, before and after the systemic or intratympanic administration of selective pharmacological antagonists. Using a normalized coefficient of variation $\left(\mathrm{CV}^{*}\right)$, we classified afferents as regularly $\left(\mathrm{CV}^{*}<0.1\right)$ or irregularly discharging $\left(\mathrm{CV}^{*}>0.1\right)$ and characterized their responses to either midline or ipsilateral efferent stimulation.
Comparable to previous studies in squirrel monkey and chinchilla, afferent responses to efferent stimulation were almost invariably excitatory, grew in amplitude with increasing $\mathrm{CV}^{*}$, and consisted of both fast and slow components. Efferent-mediated fast excitation was easily distinguished from slow excitation in estimates of rise time and post-stimulus duration. Large, efferent-mediated fast excitation in irregular afferents required ipsilateral EVS stimulation suggesting that ipsilateral efferent neurons preferentially innervate distinct neuroepithelial regions. Consistent with the projection of efferent neurons to the vestibular periphery, afferent excitation during EVS stimulation was abolished by sectioning the brainstem between the stimulating and recording electrodes.

Administration of the muscarinic ACh receptor (mAChR) antagonists, atropine and scopolamine blocked efferent-mediated slow excitation whereas the nicotinic $\mathrm{ACh}$ receptor (nAChR) antagonist DH E selectively blocked efferent-mediated fast excitation, while leaving the efferent-mediated slow component intact. Administration of the KCNQ potassium channel antagonist XE991 mimicked and occluded efferent-mediated slow excitation suggesting a role for an M-current mechanism in the effector pathway. Efferent-mediated inhibition of vestibular afferents were also seen in a few afferent recordings which we propose represents activation of alpha9-containing nAChRs and SK2 potassium channels in type II hair cells. Scopolamine is of particular interest as it is one of the most commonly used pharmacological agents for the prevention and treatment of motion sickness. Our data suggest that anti-motion sickness drugs like scopolamine may in fact target the peripheral EVS instead of or in addition to cholinergic mechanisms in the CNS.

Acknowledgements: This work was supported by NIH/NIDCD grant R01DC016974 


\section{Impact of Otolith Dysfunction on Postural Stability and Quality of Life: A Chronic Effects of Neurotrauma Consortium (CENC) Study}

\section{(Abstract 62, Poster Location BB)}

\author{
Courtney D. Hall, $\mathrm{PhD}^{1,2}$, Faith W. Akin, $\mathrm{PhD}^{1,2}$, \\ Owen D. Murnane, $\mathrm{PhD}^{1,2}$, Jennifer R. Sears, \\ AuD ${ }^{1,2}$, Richard B. Atlee ${ }^{1}$, Barry S. Eggleston, $\mathrm{MS}^{3}$, \\ Elizabeth V. Fogleman, BSPH ${ }^{3}$ \\ ${ }^{1}$ James H. Quillen VA Medical Center \\ ${ }^{2}$ East Tennessee State University \\ ${ }^{3} R T I$ International
}

Background: Until recently, clinical vestibular function assessment was limited to measurement of horizontal semicircular canal pathways. Vestibular evoked myogenic potentials are becoming more widely used to supplement the vestibular test battery by providing information about the otolith organs and their pathways; yet, the clinical significance of otolith organ dysfunction is unclear. The purpose of this study was to determine the functional consequences of otolith organ dysfunction on postural stability and quality of life.

Methods: A prospective case-control study of Veterans $(n=130)$ was completed. Comprehensive vestibular site-of-lesion testing was performed and participants were grouped according to patterns of vestibular test findings. Three vestibular groups included individuals complaining of dizziness/ imbalance with: (1) otolith organ dysfunction only (Otolith Only, $\mathrm{n}=21$ ), (2) otolith organ and semicircular canal dysfunction (Otolith+Canal, $\mathrm{n}=19$ ), and (3) semicircular dysfunction only (Canal Only, $\mathrm{n}=12$ ). Two control groups included individuals with normal vestibular function and (1) complaining of dizziness/imbalance (Dizzy Control, $\mathrm{n}=52$ ) or (2) with no complaints of dizziness/imbalance (Healthy Control, $\mathrm{n}=26$ ). Self-report questionnaires and physical performance measures of balance and gait assessed postural stability and quality of life. MANOVAs were performed to determine significant group differences $(p<0.05)$ for balance and gait and quality of life outcome measures. As appropriate, post hoc analyses of covariance and pairwise comparisons were performed to identify specific group differences $(\mathrm{p}<0.05)$.
Results: There were no significant group differences for age, race, ethnicity, gender or occupational status. MANOVAs indicated significant group differences for both gait and balance and quality of life measures (see Table). The Otolith+Canal group performed significantly worse than both control groups and the Otolith Only and Canal Only groups on the Sensory Organization Test. The Otolith+Canal group also performed significantly worse than both control groups on the Functional Gait Assessment. The Otolith Only group performed significantly worse than the Healthy Control group on a measure of the impact on activities, the Activities-specific Balance Confidence scale (ABC), and the Dizziness Handicap Inventory (DHI). The Otolith+Canal group performed significantly worse than the Healthy Control on a measure of the impact on activities, ABC, DHI, and Vestibular Activities and Participation measure.

Conclusions: Otolith organ dysfunction negatively impacts quality of life, and in conjunction with semicircular canal dysfunction, negatively impacts balance and gait. The findings of this study have important implications for developing effective clinical protocols for the diagnosis and management of individuals with dizziness related to otolith organ dysfunction.

\section{Biophysical Model of the Vestibular Hair Cell Synapse}

\section{(Abstract 63, Location CC)}

Aravind Chenrayan Govindaraju'; Imran Quraishi²; Anna Lysakowski³; Ruth Anne Eatock ${ }^{4}$; Robert M. Raphael ${ }^{1}$

${ }^{1}$ Rice University; ${ }^{2}$ Yale University; ${ }^{3}$ University of Illinois at Chicago; ${ }^{4}$ University of Chicago

Within the past decade, tremendous advances have been made identifying and characterizing the ion channels and pumps within the sensory epithelia of the vestibular inner ear. In mammals and other amniotes, a striking feature of these epithelia is the presence of cup-shaped synaptic terminals (calyces) made by primary afferent neurons on specialized mechanosensory cells (type I hair cells). Recent work has shown that type I hair cells transmit to calyces by two mechanisms: (1) conventional release of glutamate from vesicles ("quantal") and (2) 
unconventional flow of ions from the hair cell into the cleft and the postsynaptic calyx ("non-quantal"). Details of this complex transmission remain unknown, and the relevant compartments (cells and synaptic cleft) are hard to access, hindering the measurement of ion concentrations and potentials. The objective of this study is to create a biophysical model of the vestibular hair cell-calyx synapse (VHCC) with the goal of understanding the role of both quantal and non-quantal transmission. The VHCC model incorporates the specific location and surface density of ion channels (MET, HCN, $\mathrm{K}_{v}$, $\mathrm{Ca}_{\mathrm{v}}, \mathrm{Na}_{\mathrm{v}}$ ) and pumps (Na-K ATPase, $\mathrm{KCC}$ ) expressed in hair cells and calyces, along with measurements of cell and calyx geometry from electron micrographs. The dynamic behavior of the system is determined from measured or estimated open probabilities, conductance, and activation time constants of the channels. The VHCC model is implemented in COMSOL and Matlab using a variant of the cable equation along with $\mathrm{K}^{+}$and $\mathrm{Na}+$ electrodiffusion in the cleft, simplified HodgkinHuxley-style ion currents, and stochastic vesicle release along an axisymmetric parametric surface. After establishing steady-state conditions, the input to the model is a step deflection of the hair bundle, and the outputs include the $\mathrm{K}^{+}, \mathrm{Na}^{+}$and potential gradients within the cleft, the voltage changes on the inner surface of the calyx, and the pattern of spiking in the nerve fiber. The overall predictions are consistent with experimental recordings from the vestibular hair cell synapse, and the model is able to predict how increases in cleft $\mathrm{K}^{+}$influence the extent of non-quantal transmission. The model is also able to make quantitative predictions on how alterations in the conductance of specific channels in both the hair cell and the calyx alter the ultimate pattern of firing in the vestibular nerve. These latter predictions may provide important clues to mechanisms of hidden vestibular loss and suggest strategies for pharmacological intervention in vestibular disorders.

Acknowledgements: Funded by NIDCD R01DC012347

\section{Patients with Potential Vestibular Migraine Demonstrate Visual-Vestibular Mismatch not Visual Dependence}

\author{
(Abstract 64, Location ZZ)
}

Doaa S. Al Sharif, PT, MS and

Emily A. Keshner, PT, EdD

Department of Physical Therapy, Temple

University, Philadelphia PA

Pamela Roehm, MD, PhD

and Logan Lindemann, BS

Dept. of Otolaryngology, Temple Univ. School of

Medicine, Philadelphia PA

Dizziness affects $20-30 \%$ of the general population. A subgroup of dizzy patients with chronic migraine suffer vertigo, gaze instability, and sensitivity to visual and head motion. This disorder has been termed vestibular migraine or migrainous vertigo (1). Vestibular migraine remains a diagnosis of exclusion based on history, and many patients only receive a partial alleviation of symptoms with medication. Recent evidence suggests that dizziness and instability generated by complex visual environments (e.g., the grocery store) or with widefield of view visual motion (e.g., movie screen) results from an inability to correctly process or adapt to conflicting visual and vestibular signals. We hypothesized that dizzy individuals with headache, i.e., "Potential Vestibular Migraine (PVM)" will test positive for Visual-Vestibular Mismatch (VVM) and exhibit increased weighting (dependence) on visual information as tested on the rod-and-frame test. A convenience sample of patients presenting to the otologic clinic with a variety of vestibular complaints and diagnoses underwent visual dependence and rod-and-frame testing. Results on the Rod and Frame test were compared to results previously collected from 8 young (21-50 yrs) and 12 older (60-68 yrs) healthy adults (2). Visual-vestibular mismatch (VVM) was measured using a modified VVM questionnaire (3). Visual error to subjective visual vertical and horizontal was measured using a computerized rod-and-frame test (Virtualis, France) projected as a $3 \mathrm{D}$ virtual reality image on a headmounted display (Oculus Rift). Of the 74 men and women (M: F 22\% v. 52\%; average age 56.2 years; range 23-82 years) who presented to the Otolaryngology clinic with complaints of dizziness, 
$42(56.8 \%)$ tested positive for VVM. $68.9 \%$ of patients with headache tested positive for VVM. $41.5 \%$ of patients were found to have visual dependency. $22.2 \%$ of patients with headache had visual dependency. Patients with complaints of headache with dizziness showed far higher rates of $\operatorname{VVM}(p=0.01)$ and visual dependency $(p=0.19)$ than dizzy only patients. This suggests that reweighting of sensory stimuli occurs for patients with PVM in general. VVM occurs at even greater rates for patients with migraine, and provides future directions for novel rehabilitation therapy for these patients.

\section{References}

(1) Furman JM, Balaban CD (2015). Ann NY Acad Sci, 1343 (90).

(2) Slaboda $\mathbf{J}$ et al (2009). Ann Intern Conf IEEE EMBS, (1147).

(3) Mallinson AI, Longridge NS (2005). Otol Neurotol, 26:686-91.

Acknowledgments: Funding for Ms. Al Sharif comes from the Saudi Arabian Cultural Mission in United States. Software was provided by Virtualis: www.VirtualisVR.com. 